\title{
LOS ESCARABEIDOS Y GEOTRÚPIDOS DE LA COMUNIDAD DE MADRID: LISTA DE ESPECIES, DISTRIBUCIÓN GEOGRÁFICA Y PATRONES DE DIVERSIDAD (COLEOPTERA, SCARABAEOIDEA, SCARABAEIDAE Y GEOTRUPIDAE)
}

\author{
J. M. Lobo ${ }^{1}$ y J. Hortal ${ }^{1,2}$
}

\begin{abstract}
RESUMEN
En este trabajo se resumen las características fundamentales de la fauna de Geotrupidae y Scarabaeidae (Coleoptera, Scarabaeoidea) que habita la Comunidad de Madrid, presentándose un inventario actualizado así como mapas que representan la distribución observada y potencial de todas las especies. Finalmente, se estima la variación geográfica de la riqueza de especies, la rareza o la endemicidad, discutiendo brevemente el patrón geográfico encontrado.

Palabras clave: escarabajos coprófagos, Scarabaeidae, Geotrupidae, listado de especies, distribución, riqueza de especies, rareza, endemicidad, Península Ibérica, Comunidad de Madrid.
\end{abstract}

\begin{abstract}
The scarabeids and geotrupids of Comunidad de Madrid: species list, geographical distribution and diversity patterns (Coleoptera, Scarabaeoidea, Scarabaeidae and Geotrupidae)

In this work we summarize the main characteristics of Geotrupidae and Scarabaeidae (Coleoptera, Scarabaeoidea) fauna inhabiting Comunidad de Madrid, including an updated checklist of species as well as maps representing the observed and potential distributions of all species. Geographical variation in species richness, rarity or endemicity was also estimated, with a brief discussion on the geographical pattern obtained.

Key-words: dung beetles, Scarabaeidae, Geotrupidae, checklist, species distribution, species richness, rarity, endemicity, Iberian Peninsula, Comunidad de Madrid.
\end{abstract}

\section{Antecedentes}

Escarabeidos y Geotrúpidos son dos familias con grados de conocimiento taxonómico y corológico diferentes, como lo son también, parcialmente, sus hábitos e historia evolutiva. Gracias a la reciente obra de Martín-Piera y López-Colón (2000), en la que se recopila el conocimiento taxonómico, biogeográfico y ecológico de ambas familias en la Península Ibérica, es posible ahora ofrecer con facilidad un listado de las especies existentes en la Comunidad de Madrid. Sin embargo esta obra solo

Departamento de Biodiversidad y Biología Evolutiva. Museo Nacional de Ciencias Naturales (CSIC). C/José Gutiérrez Abascal, 2; Madrid - 28006; Spain

2 Departamento de Ciências Agrárias, CITA-A. Universidade dos Açores. Campus de Angra, Terra-Chã; Angra do Heroísmo - 9701-851 - Terceira (Açores); Portugal 
Tabla 1.- Principales datos de las 15 especies de la familia Geotrupidae presentes en la Comunidad de Madrid. Número de registros $(R)$, número de individuos $(N)$ y número de cuadrículas UTM de $100 \mathrm{~km}^{2}(C)$ en las que aparece cada especie.

Table 1.- Main data on the 15 species of Geotrupidae present in Comunidad of Madrid. Number of records $(R)$, individuals $(N)$ and number of $100 \mathrm{~km}^{2}$ UTM cells (C) in which each species occurs.

\begin{tabular}{|c|c|c|c|c|}
\hline Especie & Distribución geográfica & $\boldsymbol{R}$ & $N$ & $C$ \\
\hline Bolbelasmus bocchus (Erichson, 1841) & Ibero-magrebí & 1 & 2 & 1 \\
\hline Bolbelasmus gallicus (Mulsant, 1842) & Mediterráneo Occidental & 23 & 130 & 5 \\
\hline Typhaeus (Typhaeus) typhoeus (Linnaeus, 1758) & Europea & 81 & 171 & 23 \\
\hline Ceratophyus hoffmannseggi Fairmaire, 1856 & Ibero-magrebí & 36 & 159 & 11 \\
\hline Ceratophyus martinezi Lauffer, 1909 & Ibérica & 4 & 4 & 3 \\
\hline Jekelius (Jekelius) albarracinus (Wagner, 1928) & Ibérica & 1 & 1 & 1 \\
\hline Jekelius (Jekelius) castillanus (López-Colón, 1985) & Ibérica & 14 & 21 & 7 \\
\hline Jekelius (Jekelius) nitidus (Jekel, 1866) & Ibérica & 2 & 2 & 2 \\
\hline Silphotrupes escorialensis (Jekel, 1866) & Ibérica & 140 & 226 & 25 \\
\hline Trypocopris (Trypocopris) pyrenaeus (Charpentier, 1825) & Europea & 28 & 48 & 8 \\
\hline Anoplotrupes stercorosus (Scriba, 1790) & Paleártico Occidental & 17 & 21 & 5 \\
\hline Geotrupes ibericus Baraud, 1958 & Ibérica & 88 & 328 & 19 \\
\hline Geotrupes mutator (Marsham, 1802) & Eurocaucásica & 50 & 167 & 8 \\
\hline Geotrupes stercorarius (Linnaeus, 1758) & Holártica & 34 & 62 & 10 \\
\hline Sericotrupes niger (Marsham, 1802) & Paleártico Occidental & 20 & 29 & 10 \\
\hline
\end{tabular}

esboza narrativamente la distribución general Ibérica de estas especies siendo necesario para ello recopilar todo el conjunto de información corológica existente en la bibliografía y las colecciones de historia natural. SCAMAD, una base de datos que compila esta información para la Comunidad de Madrid (Hortal, 2004) ofrece ahora esta posibilidad. Utilizando ambas fuentes de información, este trabajo ofrece un listado prácticamente definitivo de las especies de estas dos familias que habitan la Comunidad de Madrid, proporcionando además una cartografía individualizada de la distribución observada y potencial de cada una de las especies en el territorio madrileño y provincias limítrofes. El sumatorio de estos mapas individuales nos ha permitido, por último, ofrecer un panorama de la distribución de la riqueza y rareza de especies de ambas familias que será comentado en último lugar.

\section{Las especies de Geotrupidae}

Los Geotrupidae, incluyendo dentro de ellos a los Bolboceratinae (Verdú et al., 1998; Verdú et al., 2004) constituyen una de las familias más primitivas de Scarabaeoidea (Browne \& Scholtz, 1999), cuya taxonomía de alto rango y sus relaciones filogenéticas internas no están bien establecidas (ver, por ejemplo, Howden, 1982; Zunino, 1984a o Browne \& Scholtz, 1995 y 1999). Con alrededor de 650 especies de Geotrupidae descritas, en la
Península Ibérica podemos encontrar 35 especies de gran tamaño $(\approx 300 \mathrm{mg}$ de peso seco) que, a grandes rasgos pertenecen a tres grandes líneas filogenéticas con distribuciones e historias evolutivas diferentes (López-Colón, 2000): algunos representantes (3 especies) de una de las subfamilias basales distribuida por casi todas las regiones biogeográficas del planeta (Bolboceratinae), otros pocos elementos (2 especies) de la que parece ser la tribu más antigua dentro de los Geotrupinae (Chromogeotrupini), la cual posee una distribución esencialmente asiática $\mathrm{y}$, por último, un conjunto mayoritario de especies (30) pertenecientes a la tribu Geotrupini; el linaje mas moderno y diversificado de geotrupidos especializado en el consumo de las heces de herbívoros y ampliamente distribuido por la región Holártica.

En líneas generales, por tanto, la fauna Ibérica de geotrúpidos se caracteriza por el dominio de elementos con un probable origen Cretácico en las regiones templado-frías del hemisferio norte (Laurasia; ver Zunino, 1984a y Krell, 2000). Por ello, la mayor relevancia de los geotrúpidos en los ensamblajes de coleópteros coprófagos aparece en las zonas montañosas y septentrionales. Sin embargo, dentro de los Geotrupidae presentes en la Península Ibérica existe una importante excepción a esta regla: los géneros Jekelius y Thorectes. Las especies de estos géneros hermanos constituyen, sin duda, uno de los grupos de insectos ibéricos más genuinos y singulares cuya diversificación se ha producido durante el Terciario 

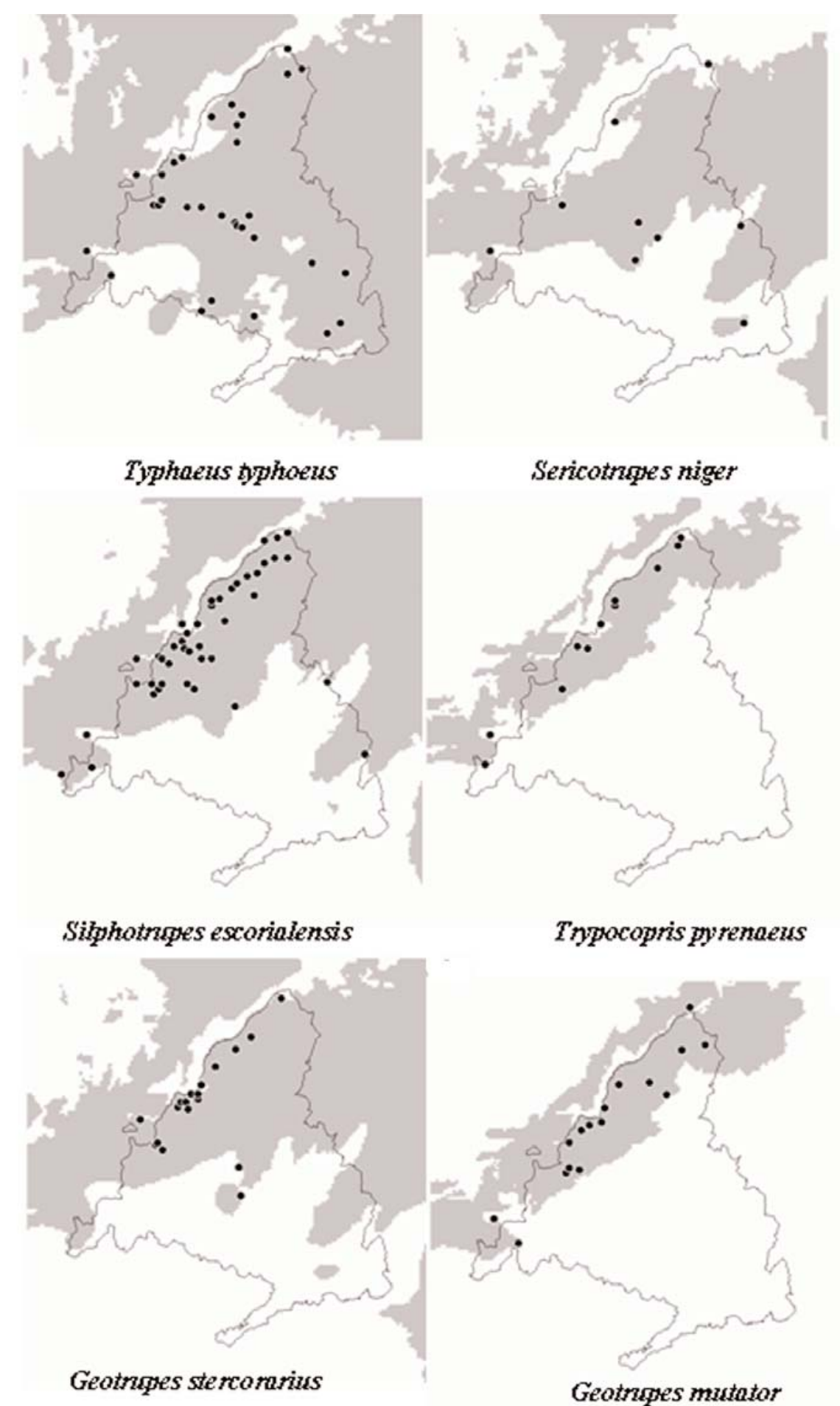

Typocopris pyrenaeus

Fig. 1.- Mapas de distribución de las especies de Geotrupidae madrileñas. Los puntos son las localidades en las que se ha observado cada especie mientras que las áreas grises representan su distribución potencial.

Fig. 1.- Distributional maps of Geotrupidae species from Madrid. Points are localities in which each species was observed while grey areas represent their potential distribution. 


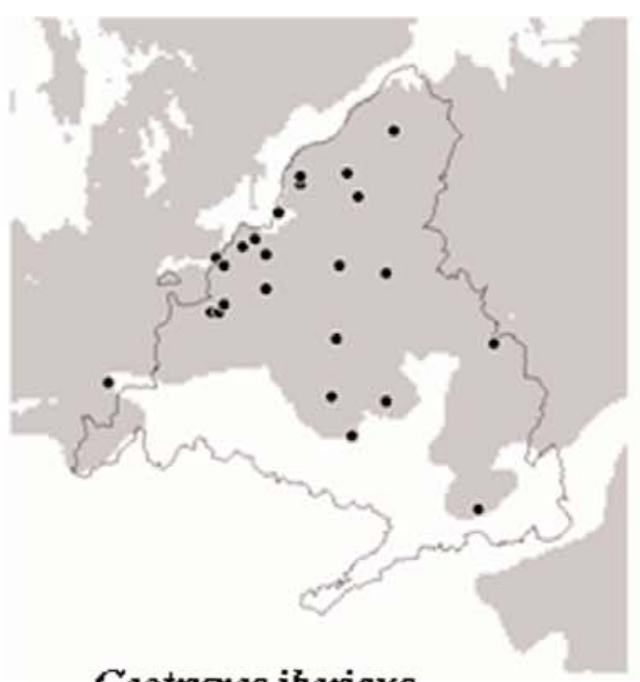

Geotnges ibericus



Jekelius albarracinus

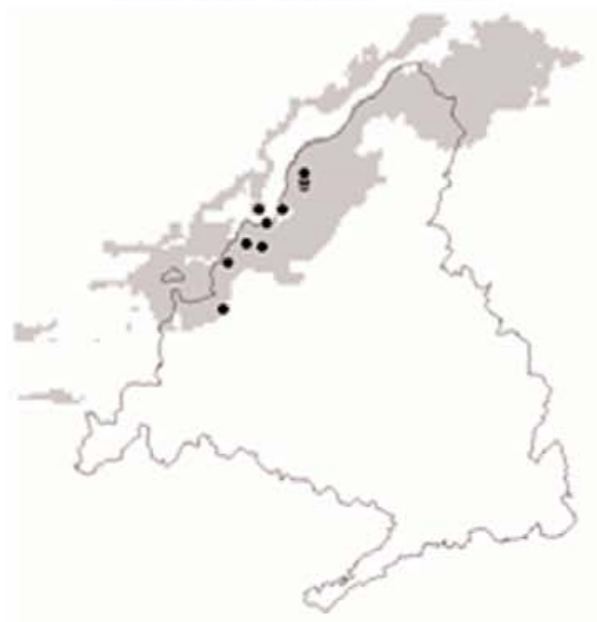

Anoplotnges stercorosus

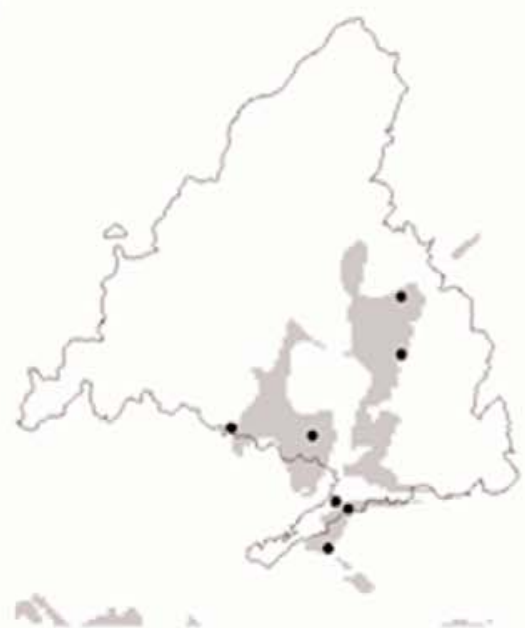

Jekelius cashillanus

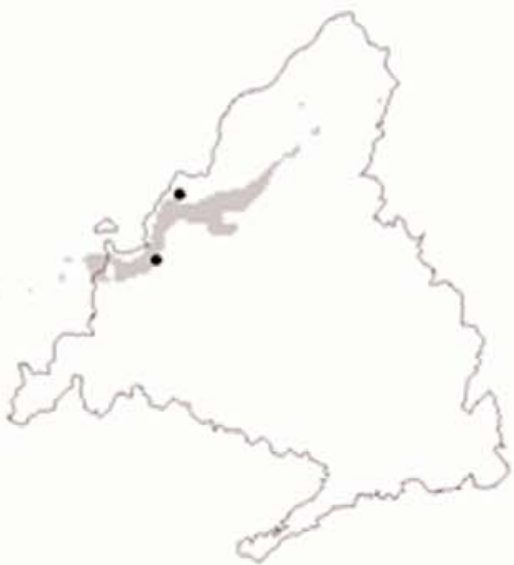

Sekelus nitidus

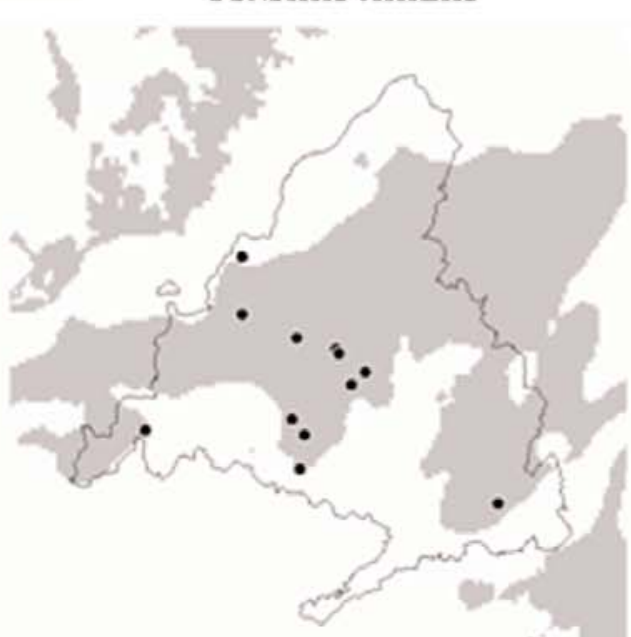

Ceratophyus hoffmannseggi

Fig. 1.-Cont. 




Ceratophyus mantinezi

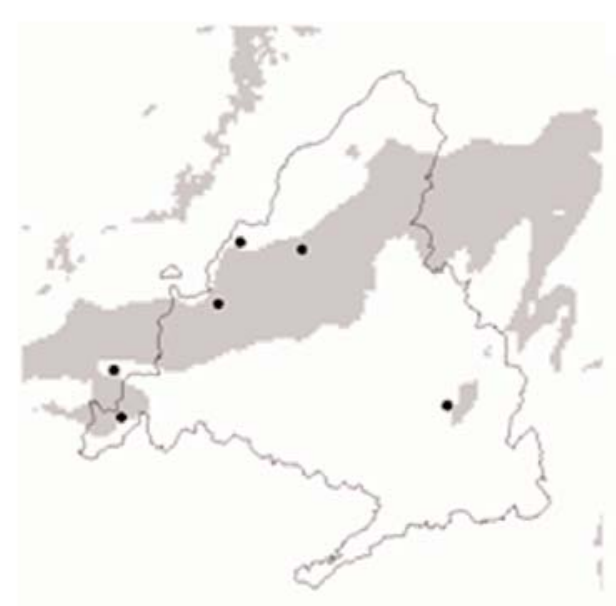

Bolbelasmus gallicus

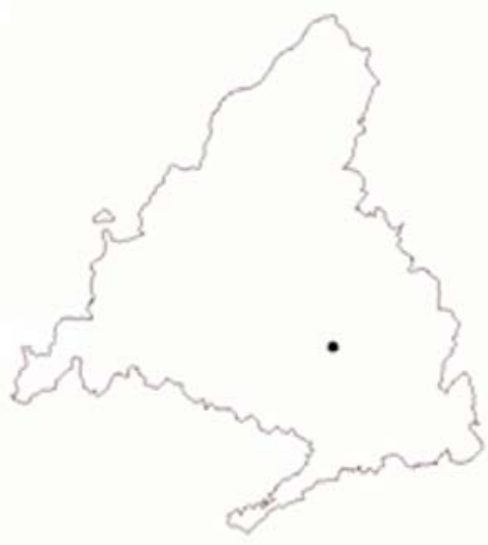

Bolbelasmus bocchus

Fig. 1.-Fin.

en la Península Ibérica mediante procesos de especiación vicariante directamente relacionados con los ciclos de conexión-disyunción entre las masas continentales africanas y mediterráneas (Palmer \& Cambefort, 1997 y 2000). Adaptadas mayoritariamente, tanto morfológica como ecológicamente, a los ambientes xéricos y al consumo de heces con bajo contenido hídrico, 17 de las 35 especies de Ibéricas de geotrúpidos pertenecen a estos géneros, influyendo decisivamente en la distribución geográfica de la endemicidad de los Scarabaeoidea Ibéricos (Verdú \& Galante, 2002) y en el hecho de que la Península Ibérica sea la región Europea con mayor número de endemismos (Lumaret \& Lobo, 1996). Sus peculiares especializaciones del aparato bucal (Verdú \&
Galante, 2004) y su apterismo, provocado probablemente ante la necesidad de evitar la pérdida hídrica mediante la fusión elytral, han sido factores claves que han limitado la capacidad de dispersión de este grupo y su alta tasa de especiación en la Península Ibérica (ver Lobo et al., 2006).

\section{LISTADO DE ESPECIES}

Familia Geotrupidae Subfamilia Bolboceratinae Mulsant, 1842 Género Bolbelasmus Boucomont, 1910

1. Bolbelasmus bocchus (Erichson, 1841)

2. Bolbelasmus gallicus (Mulsant, 1842) 

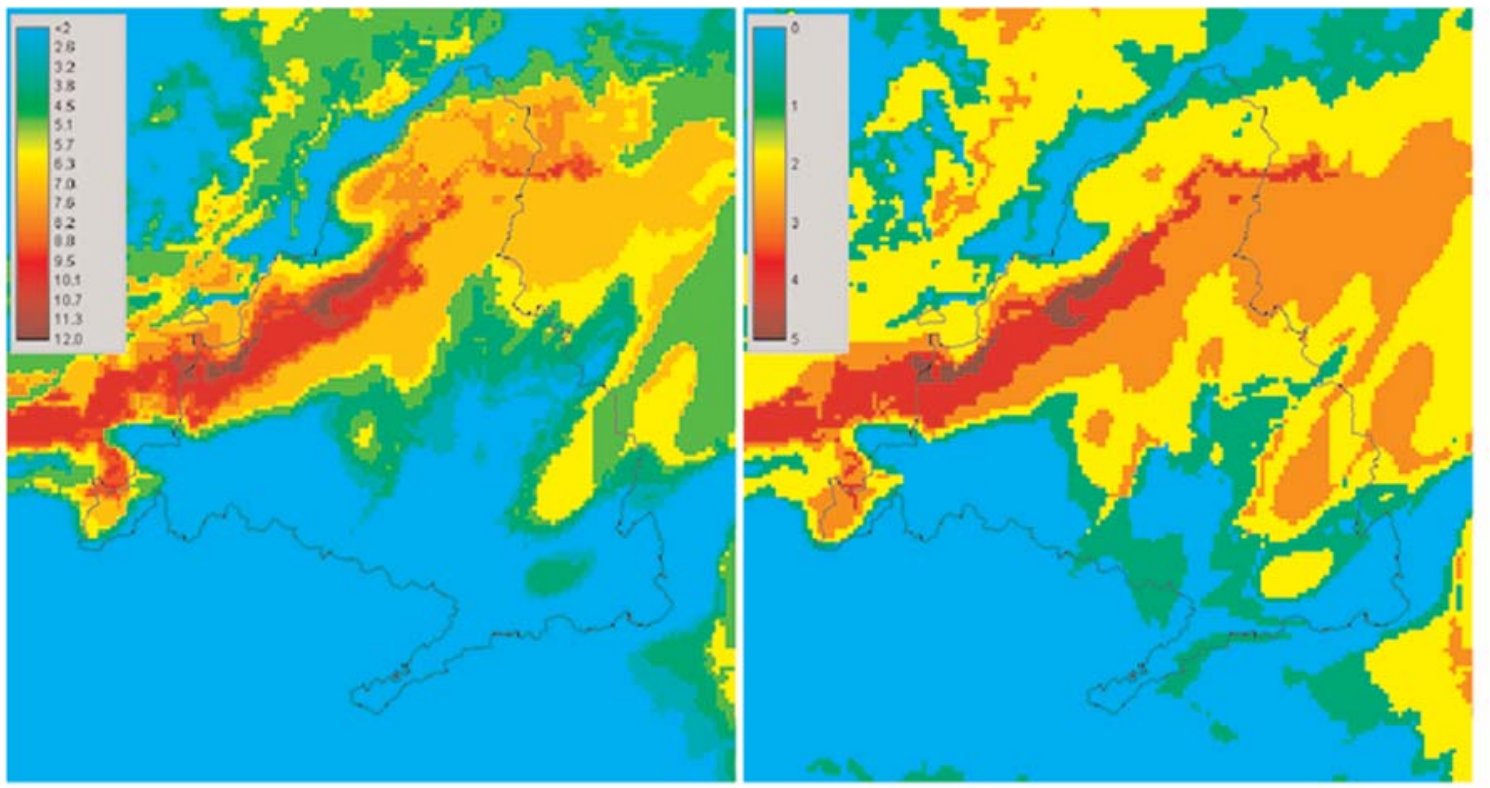

Fig. 2.- Mapas de riqueza (número de especies; izquierda) y endemicidad (número de endemismos ibéricos; derecha) para los Geotrupidae de la Comunidad de Madrid y alrededores. Cada mapa se ha obtenido mediante la superposición de las distribuciones potenciales anteriormente calculadas para todas las especies.

Fig. 2.- Maps of richness (number of species; left) and endemism (number of Iberian endemics; right) for Geotrupidae of Comunidad de Madrid and surrounding areas. Each map was obtained overlying the potential distributions previously calculated for all species.

Subfamilia Geotrupinae Latreille, 1802

Tribu Chromogeotrupini Zunino, 1984

Género Typhaeus Leach, 1815

3. Typhaeus (Typhaeus) typhoeus (Linnaeus, 1758)

Tribu Geotrupini Latreille, 1802

Género Ceratophyus Fisher de Waldheim, 1823

4. Ceratophyus hoffmannseggi Fairmaire, 1856

5. Ceratophyus martinezi Lauffer, 1909

Género Jekelius López-Colón, 1869

Subgénero Jekelius López-Colón, 1869

6. Jekelius (Jekelius) albarracinus (Wagner, 1928)

7. Jekelius (Jekelius) castillanus (López-Colón, 1985)

8. Jekelius (Jekelius) nitidus (Jekel, 1866)

Género Silphotrupes Jekel, 1866

9. Silphotrupes escorialensis (Jekel, 1866)

Género Trypocopris Motschulsky, 1858

10. Trypocopris (Trypocopris) pyrenaeus (Charpentier, 1825)

Género Anoplotrupes Jekel, 1866

11. Anoplotrupes stercorosus (Scriba, 1790)

Género Geotrupes Latreille, 1796

12. Geotrupes ibericus Baraud, 1958

13. Geotrupes mutator (Marsham, 1802)

14. Geotrupes stercorarius (Linnaeus, 1758)
Género Sericotrupes Zunino, 1984

15. Sericotrupes niger (Marsham, 1802)

\section{MAPAS DE DISTRIBUCIÓN}

Se ha realizado un mapa de distribución para cada una de las especies, incorporando todas las citas existentes en la base de datos anteriormente mencionada (SCAMAD). El área sobre la que se ha realizado esta cartografía comprende todo el territorio de la Comunidad de Madrid (unos 8.000 $\mathrm{km}^{2}$ ), pero también alrededor de $19.200 \mathrm{~km}^{2}$ pertenecientes a las provincias limítrofes de. Este área posee $160 \mathrm{~km}$ de largo por $170 \mathrm{~km}$ de ancho correspondiendo su vértices con las cuadrículas UTM de $100 \mathrm{~km}^{2}$ 30TUL56, 30TWL06, 30SUK60 y 30SWK00. La resolución utilizada para la información corológica y ambiental utilizada fue de $1 \mathrm{~km}$.

La distribución potencial de cada una de las especies fue estimada identificando el conjunto de localidades que poseían condiciones ambientales similares a las existentes en las localidades de presencia conocidas (envelope modelling; Busby, 
1986). Es decir, para cada especie se generó una hipótesis de distribución estableciendo como lugares potencialmente favorables el conjunto de las cuadrículas de $1 \mathrm{~km}^{2}$ con valores climáticos situados entre los valores máximos y mínimos de cuatro variables climáticas: precipitación total anual, precipitación durante el estío (meses Junio, Julio y Agosto), temperatura máxima del mes más calido (Julio) y temperatura mínima del mes más frío (Enero). Esta cartografía climática fue proporcionada por el Instituto Nacional de Meteorología, siendo elaborada mediante un Sistema de Información Geográfico (Idrisi Kilimanjaro; Clark Labs., 2003).

A continuación se muestran los mapas de cada una de las especies representando con puntos negros las citas conocidas y en gris el área de distribución potencial.

CARACTERÍSTICAS DE LA FAUNA MADRILEÑa Y DISTRIBUCIÓN DE LA DIVERSIDAD

Los geotrúpidos madrileños reflejan fielmente los patrones evolutivos y biogeográficos establecidos anteriormente para la fauna Ibérica. En la Comunidad de Madrid habita el $43 \%$ del total de especies Ibéricas (15 especies) y están presentes las tres líneas filogenéticas principales en las que puede subdividirse la fauna de geotrúpidos de este territorio: Bolboceratinae (2 especies), Chromogeotrupini (1 especie) y Geotrupini (12 especies). Sin embargo, debido a que las áreas áridas del sureste madrileño se encuentran en el límite septentrional de la gran superficie árida del sureste Peninsular, solamente encontramos en la Comunidad de Madrid tres especies del género Jekelius y ningún representante del género Thorectes. De este modo, aunque más de la mitad de las especies de geotrúpidos madrileñas sean endémicas de la Península Ibérica o posean una distribución Ibero-Magrebí (Tabla 1), las más abundantes y frecuentes parecen ser aquellas con una distribución mucho más amplia y templada. Podemos decir, pues, que la fauna de geotrúpidos madrileña posee un marcado carácter de transición; aunque puede encontrarse una buena representación de las principales líneas filéticas presentes en la Península Ibérica y existe una importante participación de los elementos más genuinamente ibéricos, estos últimos están básicamente relacionados con las zonas de rampa y media altura. Debido a ello, tanto el patrón de distribución del número de especies como el del número de especies endémicas es muy similar (Figura 2). En general, la rique-

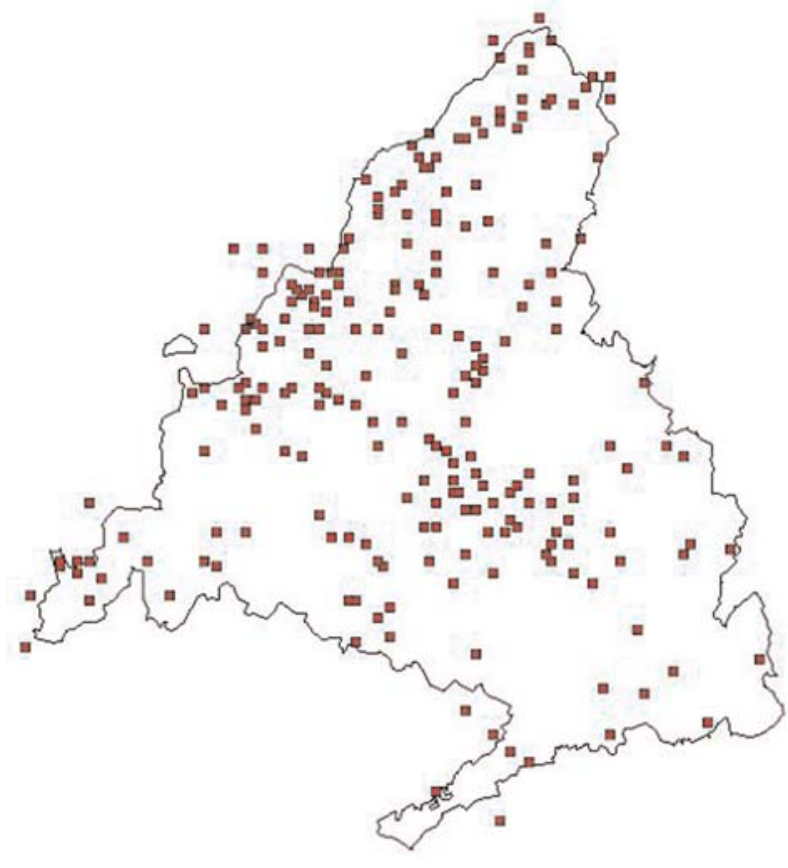

Fig. 3.- Cuadrículas UTM de $1 \mathrm{~km}^{2}$ de la Comunidad de Madrid con algún dato faunístico de Scarabaeidae.

Fig. 3. - $1 \mathrm{~km}^{2} \mathrm{UTM}$ cells of Comunidad de Madrid with some Scarabaeidae faunistic data.

za de especies es mayor en una banda noreste-suroeste que abarca la zona serrana y la rampa madrileñas hasta el sur del valle del Alberche. La endemicidad sigue este mimo patrón, aunque destaca la mayor relevancia de los páramos del sureste madrileño.

\section{Las especies de Scarabaeidae}

Esta familia agrupa a las especies con adaptaciones más especializadas hacia el consumo de las heces de herbívoro como son los cuidados parentales de los adultos hacia las larvas, los complejos sistemas de nidificación y recolocación de las heces, la oviposición de un número reducido de huevos e incluso la reducción ovárica (Halffter \& Edmonds, 1982). Con casi 300 géneros y más de 5000 especies repartidas por todas las regiones biogeográficas, los escarabeidos constituyen uno de los grupos de insectos más estudiados, cuya taxonomía y catálogo ibérico pueden considerarse bien establecidos (ver Martín-Piera 2000). En la Península Ibérica hay 55 especies de Escarabeidos, 




Scarabaeus (Ateuchetus) laticollis



Scarabaeus (Scarabaeus) sacer

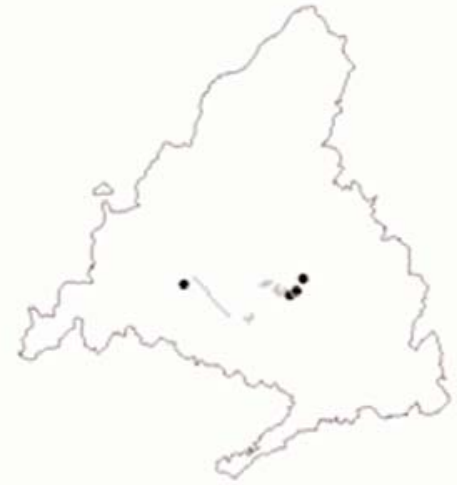

Scarabaeus (Scarabaeus) pius

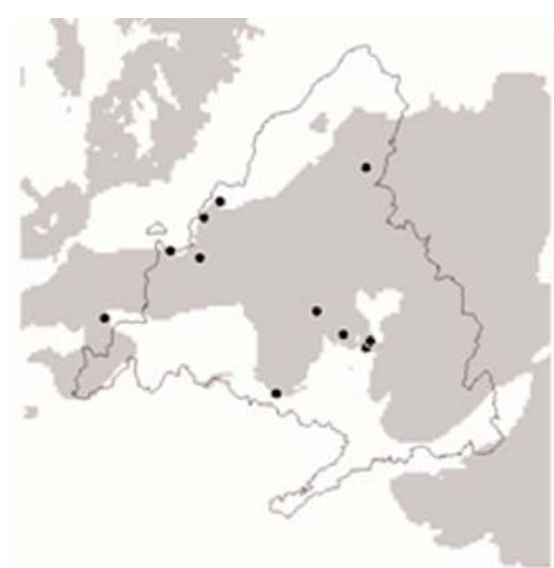

Scarabaeus (Ateuchetus) puncticollis

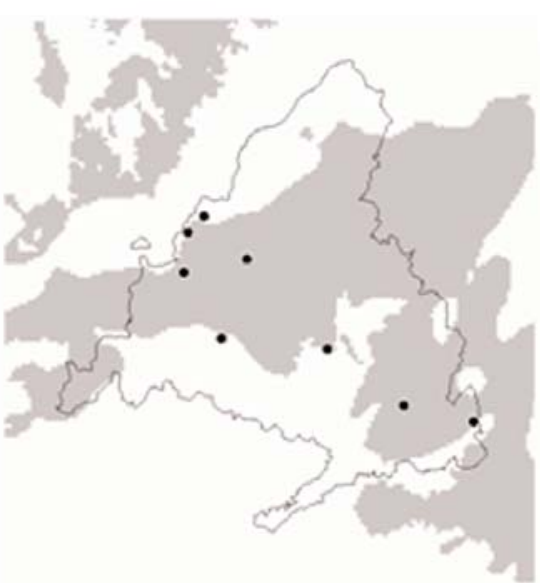

Scarabaeus (Scanabaeus) typhon

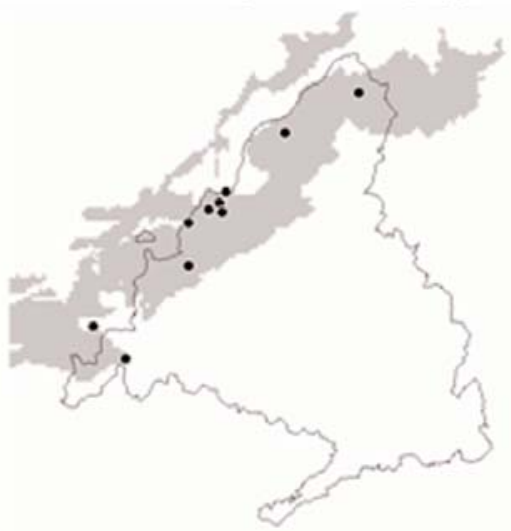

Sisphus schaefferi

Fig. 4.- Mapas de distribución de las especies de Scarabaeidae madrileñas. Los puntos son las localidades en las que se ha observado cada especie mientras que las áreas grises representan su distribución potencial.

Fig. 4.- Distributional maps of Scarabaeidae species from Madrid. Points are localities in which each species was observed while grey areas represent their potential distribution. 


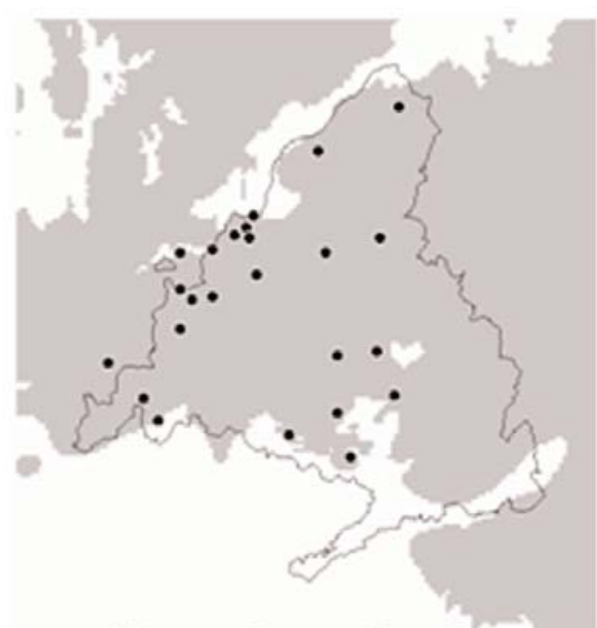

Gymnopleunis flagellatus

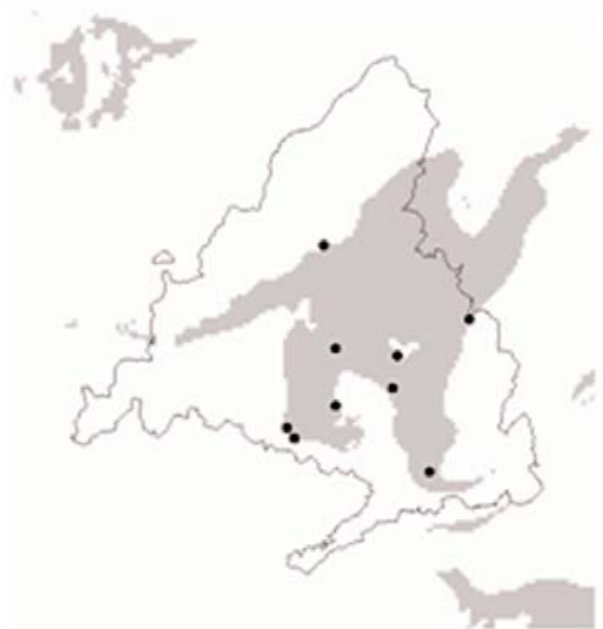

Gymnopleunus mopsus

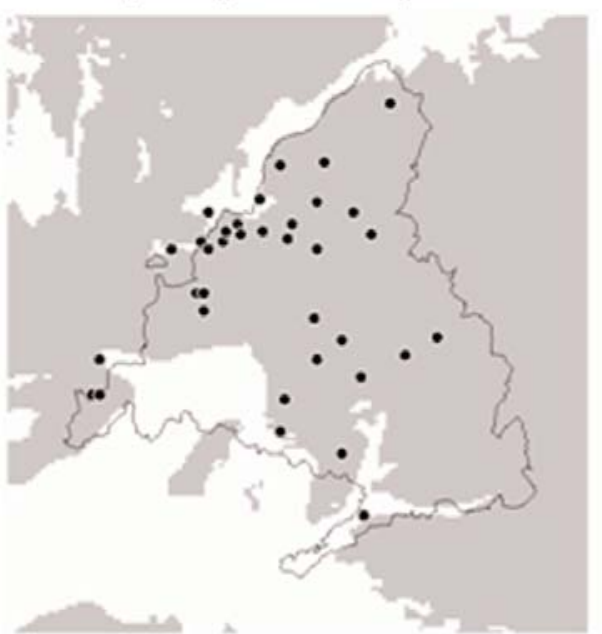

Bubas bubahs

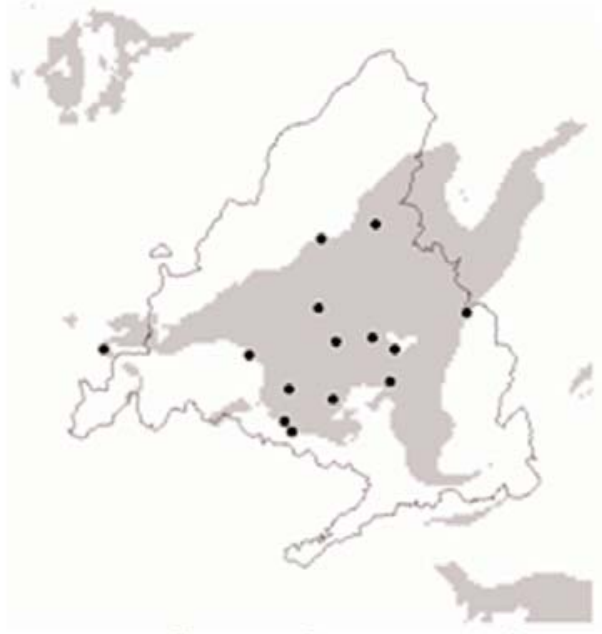

Gymnopleunus shumi

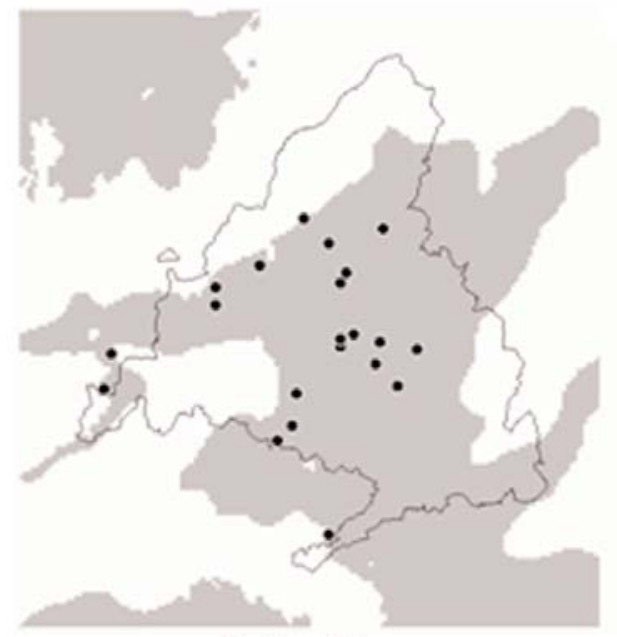

Bubas bison

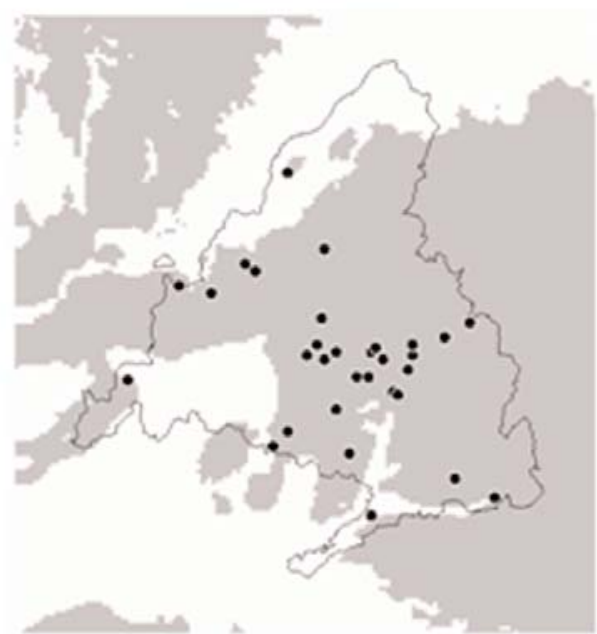

Copris hispanus

Fig. 4.-Cont. 

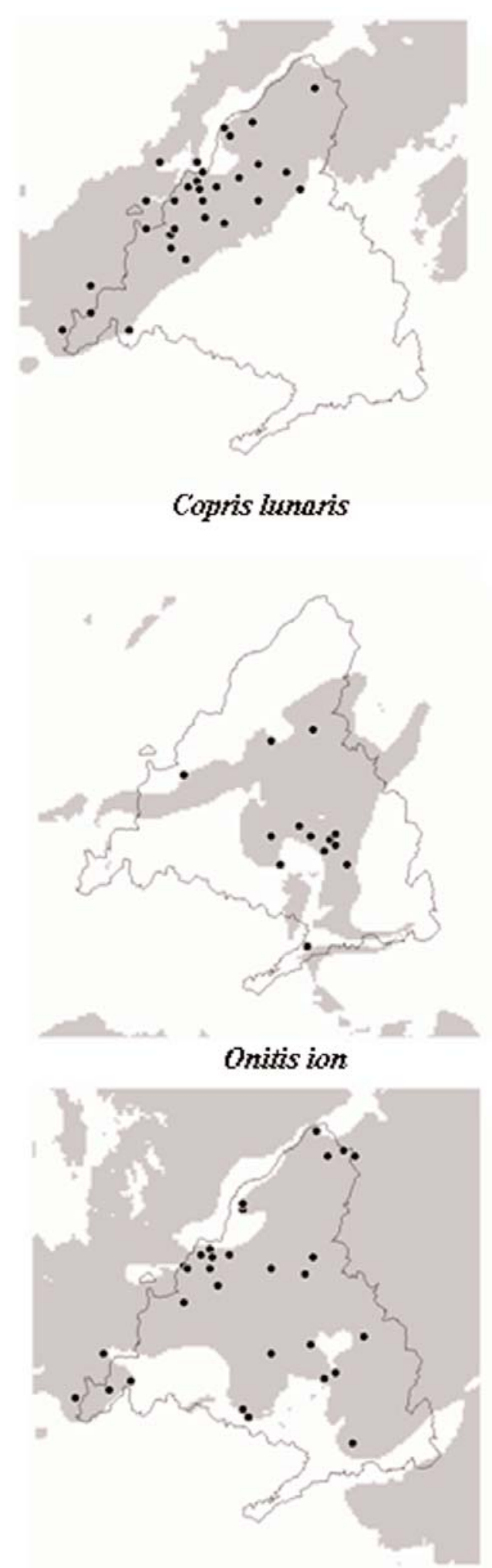

Caccobius schreber

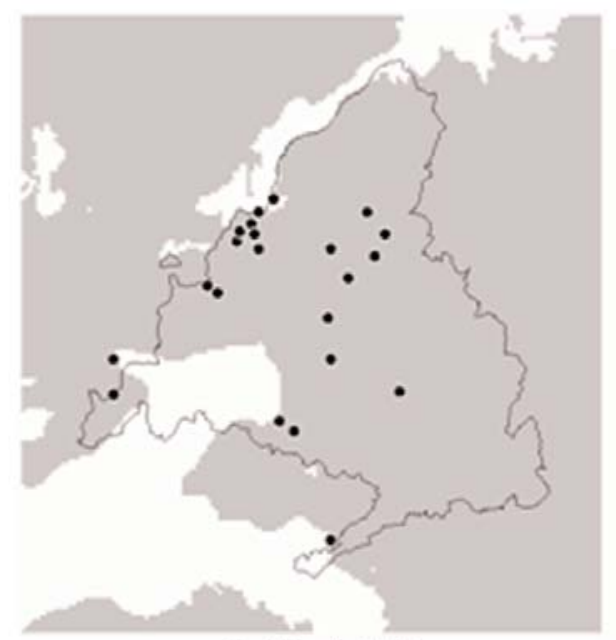

Onitis belial

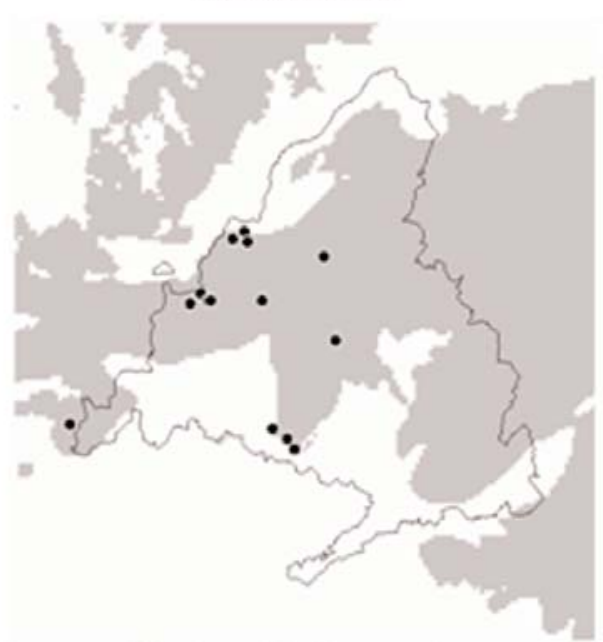

Cheironitis hungaricus

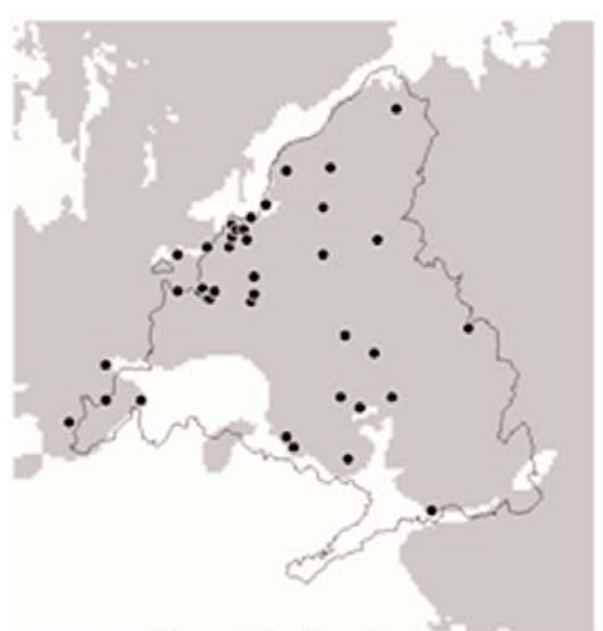

Eunoniticellus fulvus

Fig. 4.-Cont. 


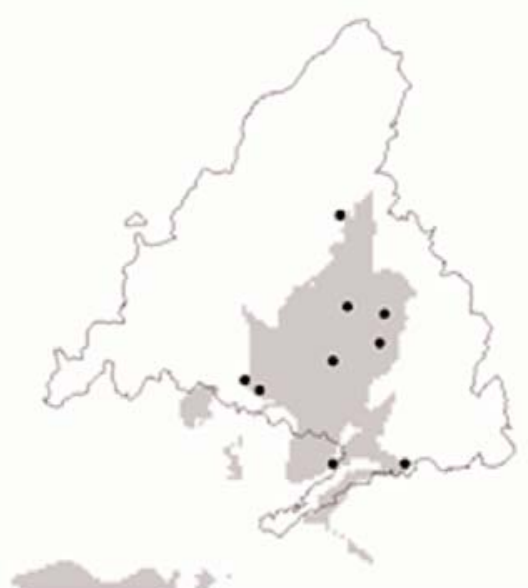

Euoniticellus pallipes



Euonthophagus amyntas

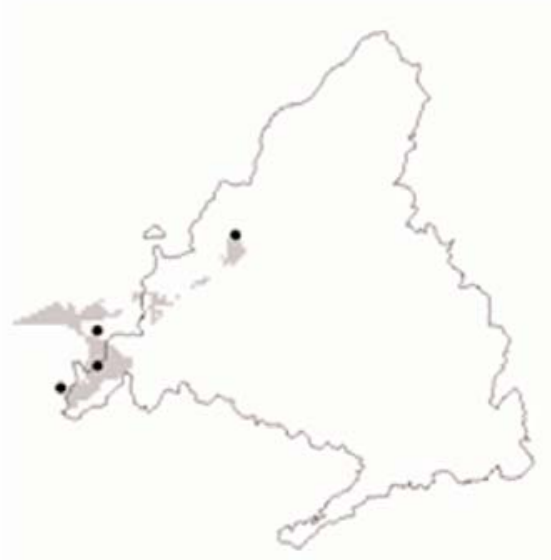

Onthophagus illyricus

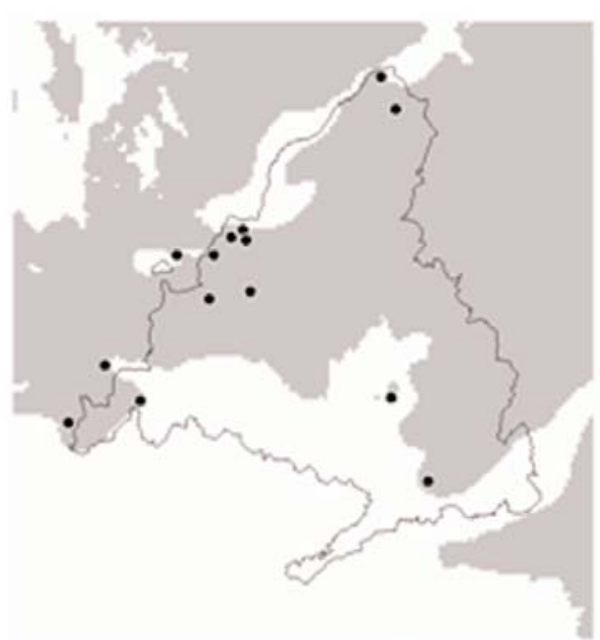

Euonthophagus gibbosus

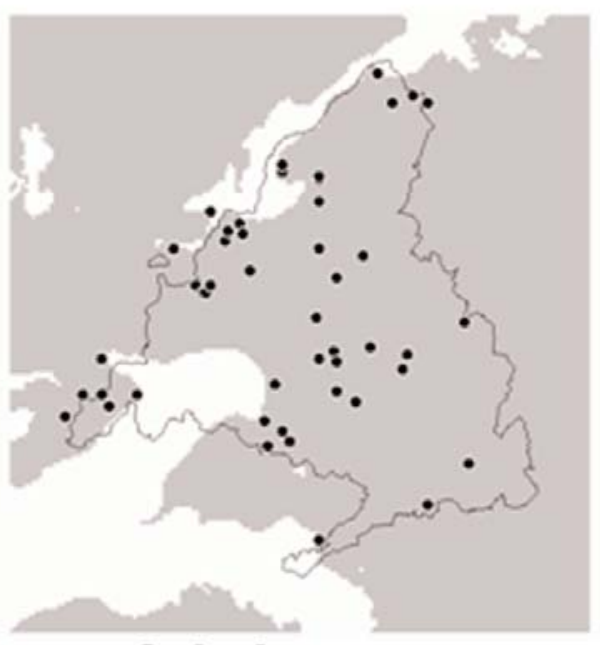

Onthophagus tauns

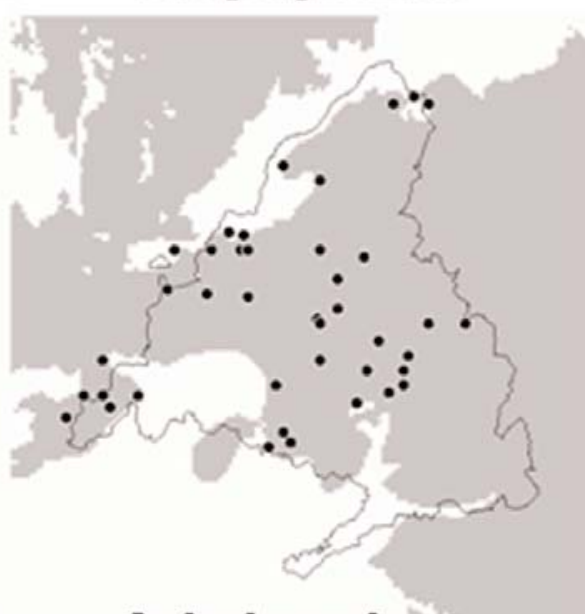

Onthophagus furcatus

Fig. 4.- Cont. 




Onthophagus sinilis

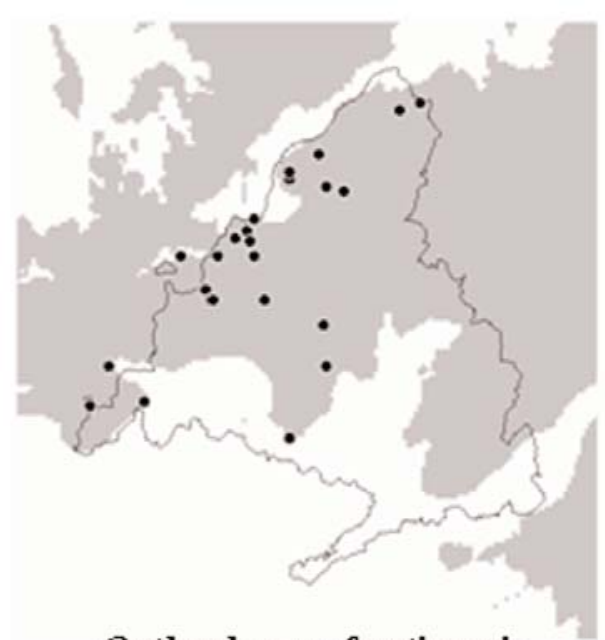

Onthophagus fracticomis

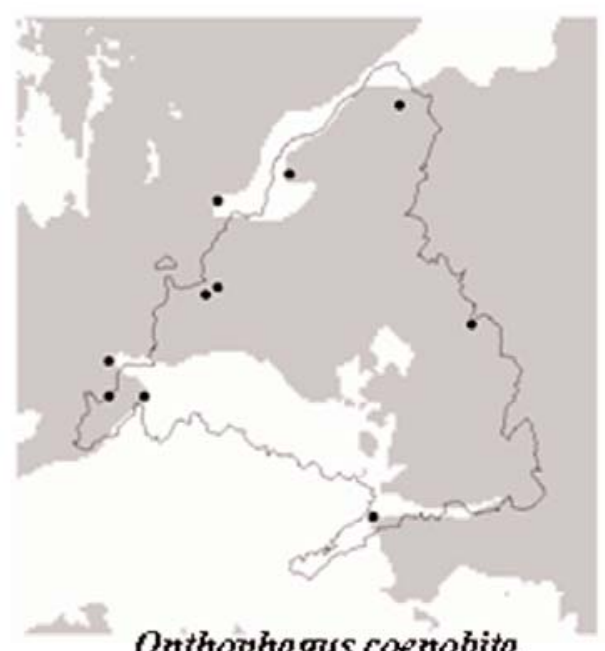

Onthophagus coenobita

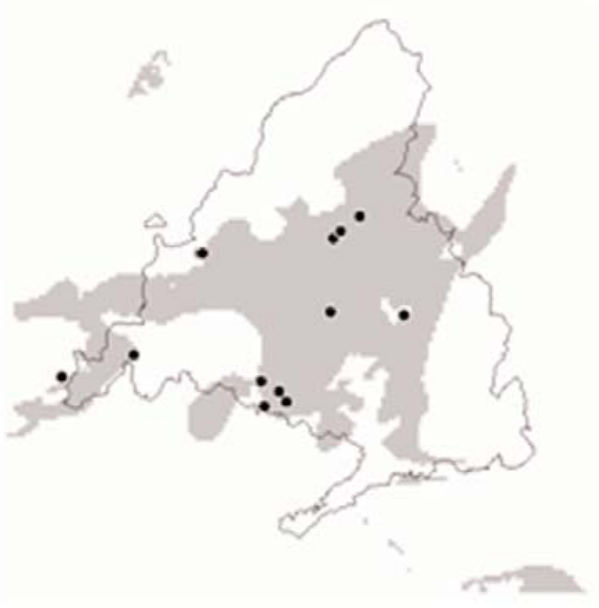

Onthophagus opacicollis

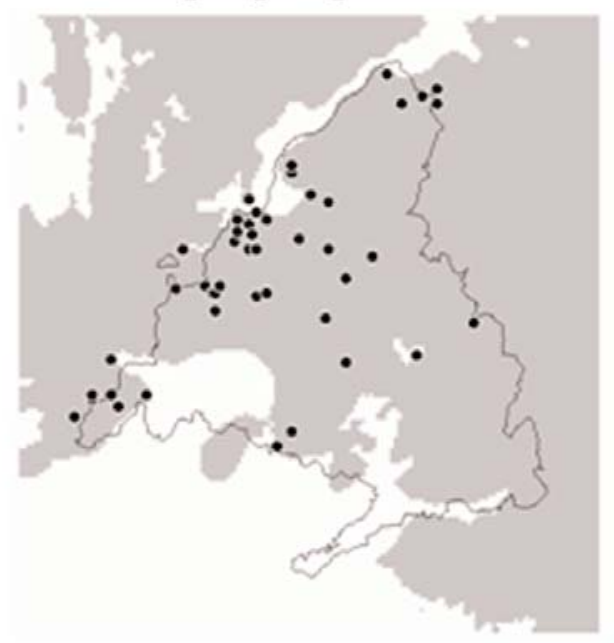

Onthophagus vacca

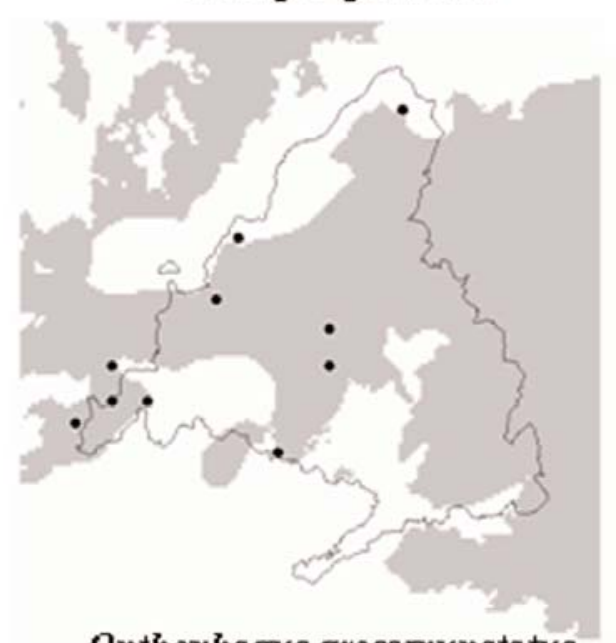

Onthophagus grosepunctatus

Fig. 4.-Cont. 


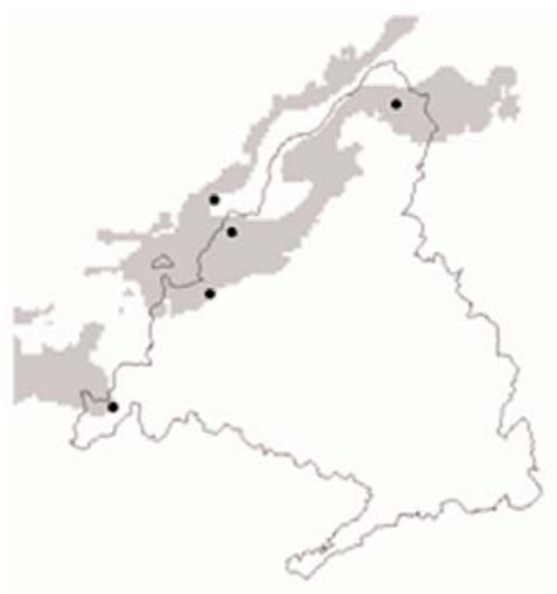

Onthophagus joannae

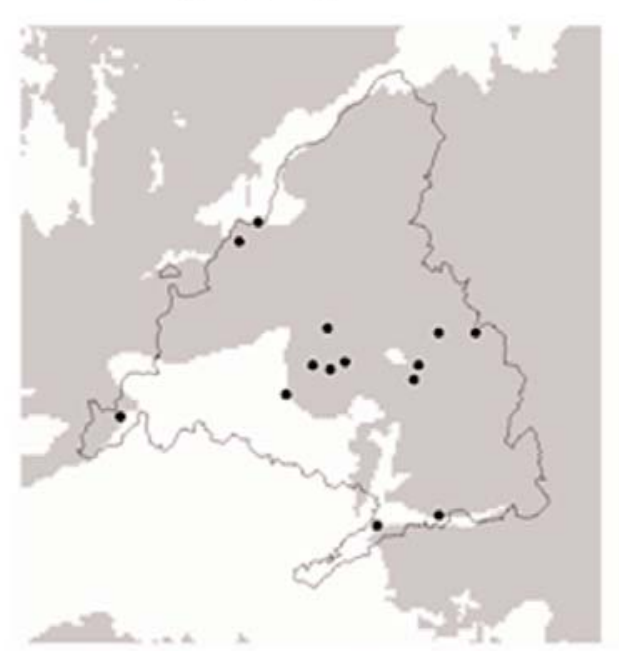

Onthophagus nuficapilles

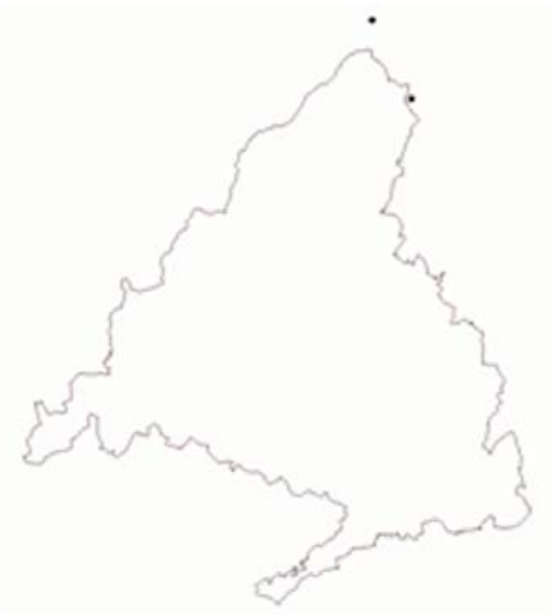

Onthophagus semicomis



Onthophagus ovatus

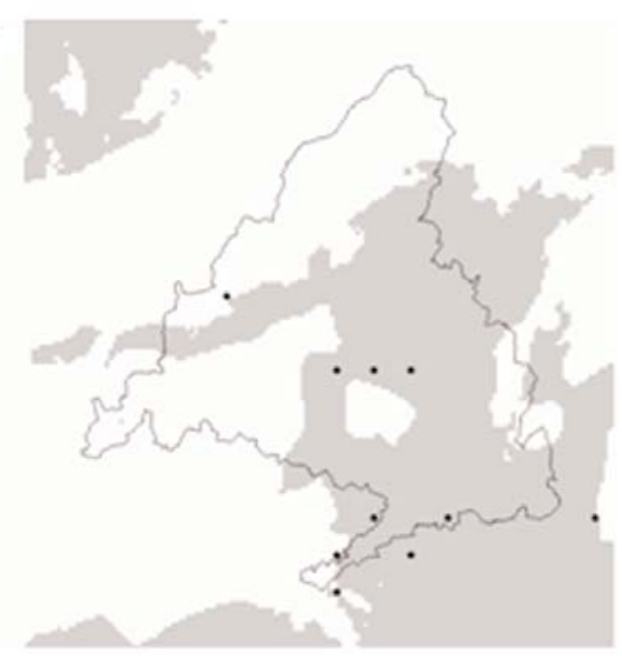

Onthophagus merdarius

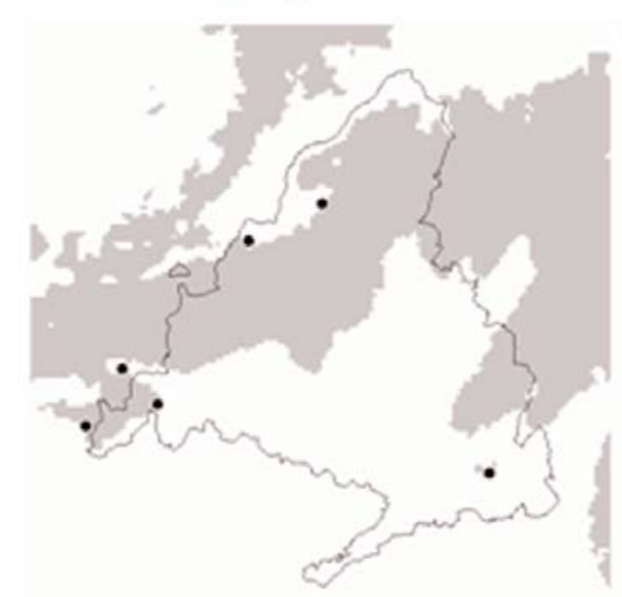

Onthophagus nutans

Fig. 4.- Cont. 




Onthophagus latigena

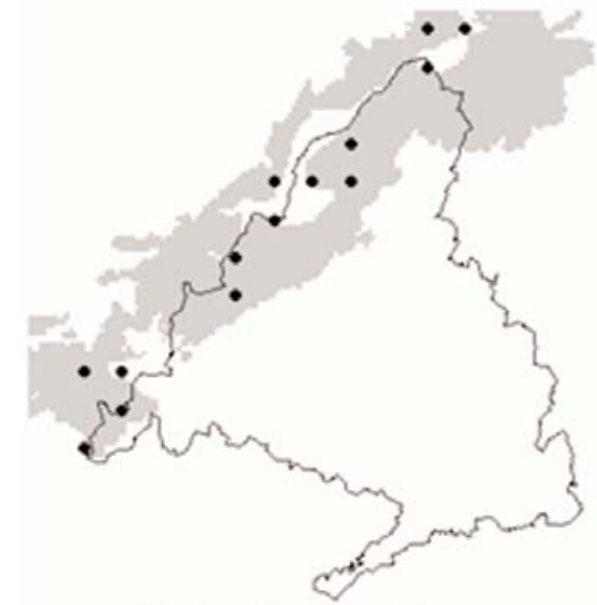

Onthophagus shlocenus

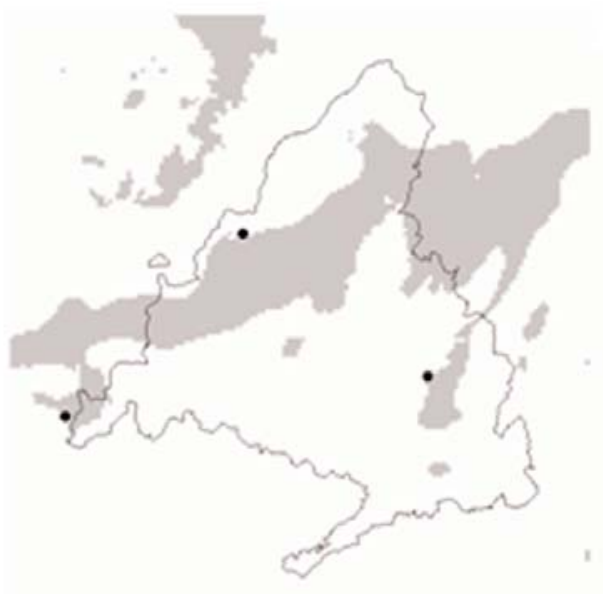

Onthophagus emarginatus

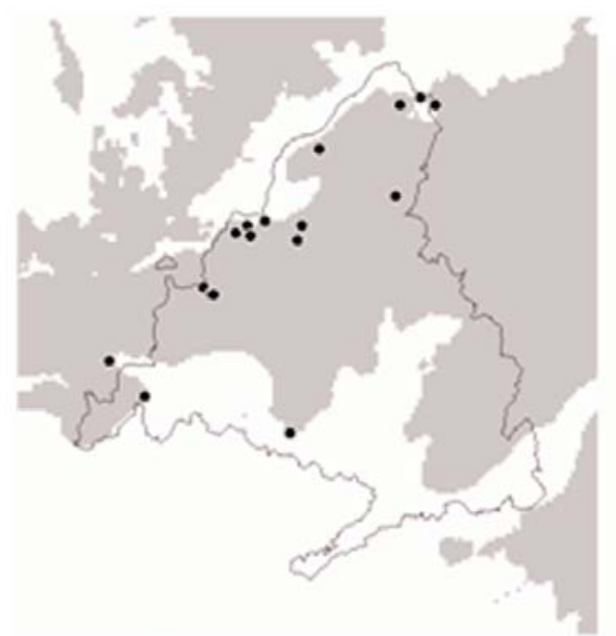

Onthophagus lemut
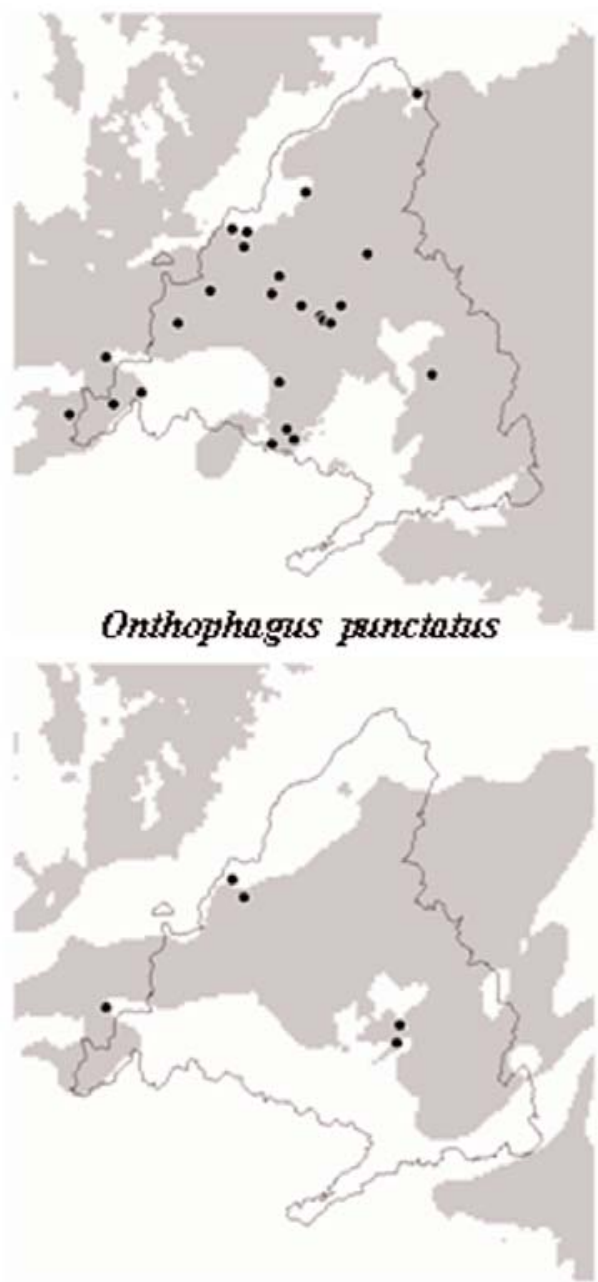

Onthophagus maki

Fig. 4.-Fin. 
Tabla 2.- Principales datos de las 42 especies de la familia Scarabaeidae presentes en la Comunidad de Madrid. Número de registros $(R)$, número de individuos $(N)$, número de cuadrículas UTM de $100 \mathrm{~km}^{2}$ de la región de estudio $(C)$ y de $2500 \mathrm{~km}^{2}$ la Península Ibérica $(\mathrm{CIb})$ en las que aparece cada especie.

Table 2.- Main data on the 42 species of Scarabaeidae present in Comunidad of Madrid. Number of records $(R)$, individuals $(N)$, number of $100 \mathrm{~km}^{2}$ UTM cells for the studied region $(C)$ and UTM cells of $2500 \mathrm{~km}^{2}$ for the Iberian Peninsula $(C I b)$ in which each species occurs.

\begin{tabular}{|c|c|c|c|c|c|}
\hline Especie & Distribución geográfica & $\boldsymbol{R}$ & $N$ & $C$ & CIb \\
\hline Sisyphus schaefferi (Linnaeus, 1758) & Paleártica & 54 & 543 & 10 & 62 \\
\hline Scarabaeus (Scarabaeus) pius (Illiger, 1803) & Medite-asiática & 12 & 18 & 4 & 5 \\
\hline Scarabaeus (Scarabaeus) sacer Linnaeus, 1758 & Medite-asiática & 14 & 14 & 13 & 80 \\
\hline Scarabaeus (Scarabaeus) typhon (Fischer de Waldheim, 1824) & Medite-asiática & 10 & 11 & 8 & 23 \\
\hline Scarabaeus (Ateuchetus) laticollis Linnaeus, 1767 & Mediterránea & 29 & 33 & 12 & 98 \\
\hline Scarabaeus (Ateuchetus) puncticollis (Latreille, 1819) & Mediterránea & 30 & 46 & 10 & 33 \\
\hline Gymnopleurus flagellatus (Fabricius, 1787) & Paleártica & 63 & 150 & 22 & 107 \\
\hline Gymnopleurus mopsus (Pallas, 1781) & Paleártica & 14 & 19 & 8 & 39 \\
\hline Gymnopleurus sturmi MacLeay, 1821 & Mediterránea & 23 & 31 & 14 & 73 \\
\hline Cheironitis hungaricus (Herbst, 1790) & Medite-asiática & 49 & 197 & 10 & 40 \\
\hline Bubas bison (Linnaeus, 1767) & Mediterránea & 68 & 376 & 19 & 81 \\
\hline Bubas bubalus (Olivier, 1811) & Mediterránea & 126 & 324 & 28 & 101 \\
\hline Onitis belial Fabricius, 1798 & Med-Occidental & 69 & 200 & 16 & 73 \\
\hline Onitis ion (Olivier, 1789) & Med-Occidental & 36 & 60 & 10 & 59 \\
\hline Copris hispanus (Linnaeus, 1764) & Med-Occidental & 76 & 141 & 22 & 109 \\
\hline Copris lunaris (Linnaeus, 1758) & Paleártica & 133 & 339 & 24 & 86 \\
\hline Euoniticellus fulvus (Goeze, 1777) & Paleártica & 128 & 1767 & 26 & 121 \\
\hline Euoniticellus pallipes (Fabricius, 1781) & Paleártica-occid & 19 & 38 & 8 & 53 \\
\hline Caccobius schreberi (Linnaeus, 1758) & Europea & 74 & 291 & 24 & 110 \\
\hline Euonthophagus amyntas (Olivier, 1789) & Europea & 70 & 180 & 19 & 108 \\
\hline Euonthophagus gibbosus (Scriba, 1790) & Europea & 26 & 49 & 14 & 34 \\
\hline Onthophagus (Onthophagus) illyricus (Scopoli, 1763) & Euroasiática & 11 & 41 & 4 & 31 \\
\hline Onthophagus (Onthophagus) taurus (Schreber, 1759) & Euroasiática & 128 & 421 & 32 & 170 \\
\hline Onthophagus (Furconthophagus) furcatus (Fabricius, 1781) & Paleártica & 118 & 2414 & 29 & 134 \\
\hline Onthophagus (Parentius) emarginatus Mulsant, 1842 & Mediterránea & 11 & 35 & 3 & 27 \\
\hline Onthophagus (Parentius) punctatus (Illiger, 1803) & Ibérica & 84 & 386 & 19 & 86 \\
\hline Onthophagus (Trichonthophagus) maki (Illiger, 1803) & Med-Occidental & 10 & 23 & 7 & 68 \\
\hline Onthophagus (Palaeonthophagus) coenobita (Herbst, 1783) & Euroasiática & 20 & 71 & 9 & 22 \\
\hline Onthophagus (Palaeonthophagus) fracticornis (Preyssler, 1790) & Europea & 87 & 326 & 18 & 76 \\
\hline Onthophagus (Palaeonthophagus) grossepunctatus Reitter, 1905 & Euro-Medite. & 41 & 585 & 10 & 57 \\
\hline Onthophagus (Palaeonthophagus) joannae Goljan, 1953 & Euro-Medite. & 11 & 14 & 6 & 47 \\
\hline Onthophagus (Palaeonthophagus) latigena d'Orbigny, 1897 & Ibero-Magrebí & 40 & 201 & 10 & 28 \\
\hline Onthophagus (Palaeonthophagus) lemur (Fabricius, 1781) & Euroasiática & 34 & 126 & 13 & 88 \\
\hline Onthophagus (Palaeonthophagus) merdarius Chevrolat, 1865 & Ibérica & 9 & 16 & 5 & 24 \\
\hline Onthophagus (Palaeonthophagus) nutans (Fabricius, 1787) & Euro-Medite. & 33 & 246 & 7 & 31 \\
\hline Onthophagus (Palaeonthophagus) opacicollis Reitter, 1893 & Mediterránea & 34 & 66 & 7 & 77 \\
\hline Onthophagus (Palaeonthophagus) ovatus (Linnaeus, 1767) & Euro-Medite. & 4 & 4 & 4 & 43 \\
\hline Onthophagus (Palaeonthophagus) ruficapillus Brullé, 1832 & Euro-Medite. & 36 & 85 & 11 & 79 \\
\hline Onthophagus (Palaeonthophagus) semicornis (Panzer, 1798) & Euro-Medite. & 1 & 2 & 1 & 6 \\
\hline Onthophagus (Palaeonthophagus) similis (Scriba, 1790) & Europea & 188 & 3253 & 32 & 128 \\
\hline Onthophagus (Palaeonthophagus) stylocerus Graells, 1851 & Ibérica & 72 & 246 & 16 & 25 \\
\hline Onthophagus (Palaeonthophagus) vacca (Linnaeus, 1767) & Paleártica & 163 & 930 & 28 & 148 \\
\hline
\end{tabular}

la última de las cuales fue descrita por Jacques Baraud hace ya 26 años.

A pesar del probable origen común de todos los Escarabeidos (Browne \& Scholtz, 1998; Scholtz \& Chown, 1995), no existe una propuesta consensuada sobre las relaciones filogenéticas de las categorías taxonómicas en las que se subdividen (subfamilias, tribus o subtribus; ver Halffter \& Edmonds, 1982;
Zunino 1984b y 1985; Cambefort, 1991; Montreuil, 1998 o Browne \& Scholtz, 1998). Los trabajos filogenéticos mas recientes, basados tanto en datos morfológicos (Philips et al., 2004a; Forgie et al., 2005) como moleculares (Villalba et al., 2002), confirman que la división clásica de esta familia en Scarabaeinae y Coprinae, basada principalmente en el tipo de nidificación y en el manejo del excremen- 
to (rodadores los primeros, tuneleadores los segundos), carece de sentido filogenético. Tampoco las relaciones entre algunas de las tribus clásicas parecen soportarse ante las nuevas evidencias filogenéticas (ver también Philips et al., 2002 y 2004b).

Actualmente se reconoce que los Escarabeidos se originaron durante el Mesozoico en el por entonces continente meridional de Gondwana (Davis et al. 2002), probablemente consumiendo heces de grandes saurios (Chin \& Gill, 1996). La actual fauna existente en las región Paleártica seria el resultado de la reciente colonización (PlioPleistocénica) de algunas especies pertenecientes a líneas Afrotropicales y de la diversificación ocurrida durante el Cenozoico de algunos taxa de origen Afrotropical, en respuesta a la radiación de los mamíferos placentarios y la expansión de los biomas herbáceos. Esta colonización y radiación en el Paleártico probablemente ocurrió durante el Mioceno y significó la generación de algunos endemismos a nivel genérico y específico (Davis \& Scholtz, 2001; Davis et al. 2002; ver también Zunino 1984b, Cambefort, 1991 y Martín-Piera, 2000). De este modo, la actual fauna Paleártica sería principalmente el resultado de la diversificación en regiones asiáticas de antiguas líneas africanas, posteriormente diezmadas como consecuencia de los cambios climáticos Cuaternarios. Por este motivo, la riqueza de géneros y especies de Escarabeidos en el Paleártico (17 y 335) es significativamente menor que la existente en la región Afrotropical (122 y 2214, respectivamente) y su composición comprende tanto tribus eminentemente africanas (Gymnopleurini, Scarabeini y Onitini) como tribus más modernas con mayor (Coprini, Oniticellini y Onthophagini) o menor (Sisyphini) representación en las regiones Paleártica y Oriental. En todo caso, no hay representación en la región Paleártica de las tribus Gondwanicas más antiguas.

La fauna Ibérica de Escarabeidos posee 55 de las 335 especies presentes en el Paleártico, pero 11 de los 17 géneros que pueden encontrarse en esta región (Martín-Piera, 2000). Estos géneros pueden dividirse en tres grupos según su origen y distribución. En primer lugar, existe un género cosmopolita (Onthophagus) que comprende la mayoría de las especies Ibéricas (28 especies) y cuya división interna en ocho linajes o subgéneros (Zunino, 1979 y Martín-Piera 2002) parece estar, a grandes rasgos, avalada por datos moleculares recientes (Villalba et al., 2002). Un segundo conjunto de géneros (Scarabaeus, Gymnopleurus, Sisyphus, Copris, Euniticellus, Caccobius, Euonthophagus y Onitis) poseen pocas especies en nuestra Península pero están bien representados en la región Afrotropical (22 especies). La amplia distribución de estas especies y la ausencia de endemismos en estos géneros sugieren que estas especies ibéricas serían emisarios de líneas que encuentran en la región Paleártica su límite septentrional de distribución. Por último, un conjunto de especies pertenecientes a géneros poco diversificados y esencialmente Paleomediterráneos (Bubas y Cheironitis; 4 especies) probablemente originados en la región Paleártica durante el Terciario.

\section{LISTADO DE ESPECIES}

En la Comunidad de Madrid pueden hallarse 42 de las 55 especies Ibéricas y todos los géneros y subgéneros de Escarabeidos presentes en la Península (excepto Onthophagus (Amphionthophagus), un subgénero no sustentado por las evidencias moleculares recientes). De este modo, la fauna madrileña puede considerarse altamente representativa de la fauna Ibérica e incluso de la Paleártica. En el presente listado no se incluye a Euoniticellus pallens (Olivier, 1789), especie típica del litoral gaditano de la que existe un dudoso y único ejemplar depositado en el Museo Nacional de Ciencias Naturales en cuya etiqueta figura la localidad de Mostoles. Tampoco se ha considerado fiable la citas de Onthophagus (Parentius) nigellus (Illiger, 1803) procedente de Velilla de San Antonio (Martín-Piera 2002) y las antiguas citas de Onthophagus (Palaeonthophagus) marginalis Gebler, 1817 procedentes de Ribas del Jarama y Rivas-Vaciamadrid, aunque en estos dos últimos casos no sería descartable que las poblaciones de estas especies se hayan extinguido en la Comunidad de Madrid.

Familia Scarabaeidae Latreille, 1802

Tribu Scarabaeini Latreille, 1802

Género Sisyphus Latreille, 1807

1. Sisyphus schaefferi (Linnaeus, 1758)

Género Scarabaeus Linnaeus, 1758

Subgénero Scarabaeus Linnaeus, 1758

2. Scarabaeus (Scarabaeus) pius (Illiger, 1803)

3. Scarabaeus (Scarabaeus) sacer Linnaeus, 1758

4. Scarabaeus (Scarabaeus) typhon (Fischer de Waldheim, 1824)

Subgénero Ateuchetus Bedel, 1892

5. Scarabaeus (Ateuchetus) laticollis Linnaeus, 1767

6. Scarabaeus (Ateuchetus) puncticollis (Latreille, 1819)

Género Gymnopleurus Illiger, 1803

7. Gymnopleurus flagellatus (Fabricius, 1787)

8. Gymnopleurus mopsus (Pallas, 1781)

9. Gymnopleurus sturmi MacLeay, 1821 
Tribu Onitini Fabricius, 1798

Género Cheironitis Lansberge, 1875

10. Cheironitis hungaricus (Herbst, 1790)

Género Bubas Mulsant, 1842

11. Bubas bubalus (Olivier, 1811)

12. Bubas bison (Linnaeus, 1767)

Género Onitis Fabricius, 1798

13. Onitis belial Fabricius, 1798

14. Onitis ion (Olivier, 1789)

Tribu Coprini Erichson, 1847

Género Copris

15. Copris hispanus (Linnaeus, 1764)

16. Copris lunaris (Linnaeus, 1758)

Tribu Oniticellini Le Peletier y Serville, 1825

Género Euoniticellus Janssens, 1953

17. Euoniticellus fulvus (Goeze, 1777)

18. Euoniticellus pallipes (Fabricius, 1781)

Tribu Onthophagini Latreille, 1802

Género Caccobius Thompson, 1859

19. Caccobius schreberi (Linnaeus, 1758)

Género Euonthophagus Balthasar, 1959

20. Euonthophagus amyntas (Olivier, 1789)

21. Euonthophagus gibbosus (Scriba, 1790)

Género Onthophagus Latreille, 1802

Subgénero Onthophagus Latreille, 1802

22. Onthophagus (Onthophagus) illyricus (Scopoli, 1763)

23. Onthophagus (Onthophagus) taurus (Schreber, 1759)

Subgénero Trichonthophagus Zunino, 1979

24. Onthophagus (Trichonthophagus) maki (Illiger, 1803)

Subgénero Parentius Zunino, 1979

25. Onthophagus (Parentius) emarginatus Mulsant, 1842

26. Onthophagus (Parentius) punctatus (Illiger, 1803)

Subgénero Furconthophagus Zunino, 1979

27. Onthophagus (Furconthophagus) furcatus (Fabricius, 1781)

Subgénero Palaeonthophagus Zunino, 1979

28. Onthophagus (Palaeonthophagus) coenobita (Herbst, 1783)

29. Onthophagus (Palaeonthophagus) fracticornis (Preyssler, 1790)

30. Onthophagus (Palaeonthophagus) grossepunctatus Reitter, 1905

31. Onthophagus (Palaeonthophagus) joannae Goljan, 1953

32. Onthophagus (Palaeonthophagus) latigena d'Orbigny, 1897

33. Onthophagus (Palaeonthophagus) lemur (Fabricius, 1781)
34. Onthophagus (Palaeonthophagus) merdarius Chevrolat, 1865

35. Onthophagus (Palaeonthophagus) nutans (Fabricius, 1787)

36. Onthophagus (Palaeonthophagus) opacicollis Reitter, 1893

37. Onthophagus (Palaeonthophagus) ovatus (Linnaeus, 1767)

38. Onthophagus (Palaeonthophagus) ruficapillus Brullé, 1832

39. Onthophagus (Palaeonthophagus) semicornis (Panzer, 1798)

40. Onthophagus (Palaeonthophagus) similis (Scriba, 1790)

41. Onthophagus (Palaeonthophagus) stylocerus Graells, 1851

42. Onthophagus (Palaeonthophagus) vacca (Linnaeus, 1767)

\section{MAPAS DE DISTRIBUCIÓN}

Si nuestro conocimiento taxonómico sobre los Escarabeidos ibéricos puede considerarse completo, no lo es nuestro conocimiento corólogico. Únicamente el 32\% de la cuadrículas UTM Ibéricas de $2500 \mathrm{~km}^{2}$ poseen inventarios que puede considerarse bien establecidos (Lobo \& Martín-Piera, 2002) y ese porcentaje decrece hasta el $8 \%$ en el caso de las cuadrículas de $100 \mathrm{~km}^{2}$ (datos inéditos). Los problemas para obtener hipótesis de distribución fiables se ejemplifican con la situación de los Escarabeidos madrileños. Aunque se trate de un grupo de especies vistosas, aunque la alpha taxonomía este resuelta y exista una importante densidad de entomólogos, todavía no se posee ninguna información faunística del $98 \%$ del territorio madrileño (Figura 3).

Siguiendo idéntica metodología a la que se mencionó anteriormente para los Geotrupidae, se presentan ahora los mapas de cada una de las especies de Escarabeidos.

CARACTERÍSTICAS DE LA FAUNA MADRILEÑA Y DISTRIBUCIÓN DE LA DIVERSIDAD

Como ya hemos comentado la fauna de Scarabaeidae de la Comunidad de Madrid constituye una excelente representación de la Ibérica. Este hecho se corrobora porque el área de distribución Ibérica y madrileña de las especies esta altamente correlacionada (coeficiente correlacion de Spearman $=0.83 ; \mathrm{n}=42 ; \mathrm{p}<0.0001)$, lo que viene a significar que las especies más ampliamente distribuidas por la Comunidad de Madrid son aquellas 

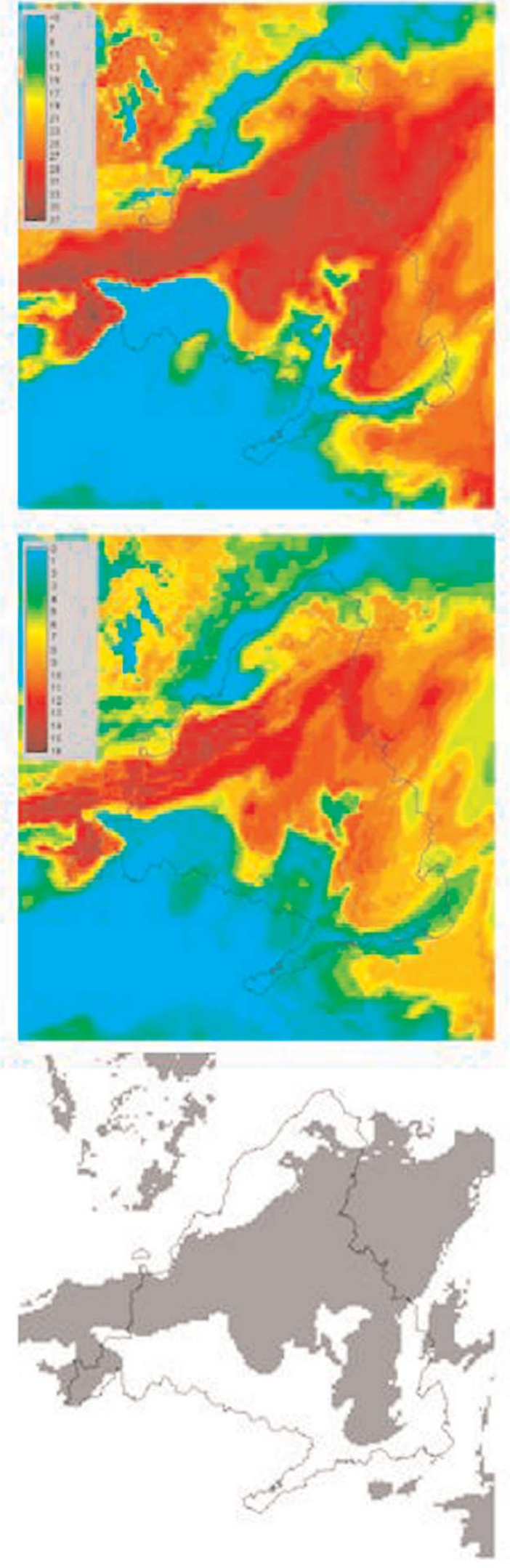

con áreas Ibéricas de distribución mayores (ver Tabla 2). En Madrid pueden encontrarse 9 de las 11 especies de escarabajos telecópridos o rodadores (los conocidos peloteros) que, como en toda la Península, están sufriendo un proceso de extinción paulatino (Lobo, 2001); pero también 4 de los 9 endemismos Ibéricos o Ibero-magrebíes existentes en la Península (Tabla 2).

Aunque más de la mitad de las especies de Escarabeidos madrileños $(57 \%$; 25 especies) poseen una distribución de tipo mediterráneo mas o menos amplia y pueden considerarse elementos adaptados a condiciones templado-cálidas, el 59\% de los registros compilados en la base de datos SCAMAD y el $77 \%$ de los especimenes pertenecen a especies con una distribución Europea, Euroasiática o Paleártica, capaces de habitar bajo condiciones templado-frías. Por ello, el número de registros, especimenes y cuadriculas en la Comunidad de Madrid difiere significativamente entre ambos tipos de especies: Mann-Whitney U test $=2.50(p<0.05 ; 2.63(p<0.01)$ y $2.72(p<0.01)$, respectivamente. De este modo, las comunidades de Escarabeidos madrileñas se caracterizan por la coexistencia de especies con distintas distribuciones y, consecuentemente, distintas adaptaciones mesoclimáticas, aunque el dominio en las comunidades parece corresponder a los elementos menos termófilos. Sólo cuatro especies de distribución Paleártica o Europea (Onthophagus similis, O. furcatus, Euoniticellus fulvus y $O$. vacca) acaparan el $58 \%$ del total de individuos colectados en la Comunidad de Madrid (Tabla 2). Por todo ello, los mapas de riqueza de especies y rareza teniendo en cuenta el número de especies con menores distribu-

Fig. 5.- Mapas de riqueza (número de especies; arriba) y rareza (número de especies Ibéricas raras; centro) para los Scarabaeidae de la Comunidad de Madrid y alrededores. Cada mapa se ha obtenido mediante la superposición de las distribuciones potenciales anteriormente calculadas para todas las especies. La rareza se ha calculado sumando el cuartil de especies presentes en un menor número de cuadrículas UTM de 50 x 50 $\mathrm{km}$ Ibéricas. El mapa de abajo representa en gris las áreas con mayores riquezas y rarezas (el $25 \%$ de los valores más elevados)

Fig. 5.- Maps of richness (number of species; up) and rarity (number of Iberian rare species; centre) for Scarabaeidae of Comunidad de Madrid and surrounding areas. Each map was obtained overlying the potential distributions previously calculated for all species. Rarity is the sum of the quartile of species present in the least number of Iberian $50 \times 50$ UTM cells. The bottom map represents in grey the areas with highest richness and rarity ( $25 \%$ of the highest scores). 
ciones Ibéricas (cuartil de las más raras; $\mathrm{n}=20$ ) son muy similares (Figura 5): los sitios más ricos en especies son generalmente aquellos en los que, al conjunto de especies de amplia distribución, se suman aquellas otras especies raras.

La riqueza media por $\mathrm{km}^{2}$ en la Comunidad de Madrid es de $17.97 \pm 0.07$ (error standard) especies (entre 37 y 0), mientras que el número de especies raras es de $5.41 \pm 0.02$ especies por $\mathrm{km}^{2}$ (entre 16 y 0 ), siendo los cuartiles superiores 27 y 9 , respectivamente (el $25 \%$ de los valores más elevados). El área por encima de estos cuartiles posee $8.216 \mathrm{~km}^{2}$ (Figura 5), una altitud media de 830 metros (entre 513 y 1669 metros), una precipitación media anual de $540 \mathrm{~mm}$ (entre 418 y $873 \mathrm{~mm}$ ) y una temperatura media anual de $12.9^{\circ} \mathrm{C}$ (entre 9.3 y $\left.14.5^{\circ} \mathrm{C}\right)$. El patrón geográfico resultante sugiere que el área de máxima diversidad para los Escarabeidos posee una forma triangular cuyos vértices se situarían, aproximadamente, en Torrelaguna, El Tiemblo y Arganda, siendo los valles del Alberche y del Tajuña los limites meridionales de esta región y las zonas serranas por encima de los 1700 metros, aproximadamente, el límite septentrional.

\section{AGRADECIMIENTOS}

Este trabajo no habría sido posible sin la ayuda de Fermín Martín Piera. Este trabajo ha sido financiado por el proyecto GR/AMB/0750/2004 de la Comunidad de Madrid. Se ha obtenido financiación adicional del proyecto de la Fundación BBVA "Yámana - Diseño de una red de reservas para la protección de la biodiversidad en América del Sur Austral utilizando modelos predictivos de distribución con taxones hiperdiversos", así como del proyecto CGL2004-0439/BOS del Ministerio de Educación y Ciencia. JH disfruta de una beca de la FCT (Fundação para a Ciência e Tecnologia) de Portugal (BPD/20809/2004).

\section{Referencias}

Browne, D. J. \& Scholtz, C. H., 1995. Phylogeny of the families of the Scarabaeoidea (Coleoptera) based on characters of the hindwing articulation, hindwing base and wing venation. Systematic Entomology, 20: 145-173.

Browne, D. J. \& Scholtz, C. H., 1998. Evolution of the scarab hindwing articulation and wing base: a contribution toward the phylogeny of the Scarabaeidae (Scarabaeoidea: Coleoptera). Systematic Entomology, 23: 307-326.

Browne, D. J. \& Scholtz, C. H., 1999. A phylogeny of the families of Scarabaeoidea (Coleoptera). Systematic Entomology, 24: 51-84.
BusBy, J. R., 1986. A biogeoclimatic analysis of Notophagus cunninghamii (Hook.) Oerst. in southeastern Australia. Australian Journal of Ecology, 11: $1-7$

CAmbefort, Y., 1991. Biogeography and evolution. In: I. Hanski \& Y. Cambefort (eds.). Dung Beetle Ecology, Princeton University Press. New Jersey: 51-67.

ChIn, K. \& Gill, B. D., 1996. Dinosaurs, dung beetles, and conifers: Participants in a Cretaceous food web. Palaios, 11: 280-285.

CLARK LABS., 2003. Idrisi Kilimanjaro. GIS software package. Clark Labs, Worcester, MA.

Davis, A. L. V. \& Scholtz, C. H., 2001. Historical versus ecological factors influencing global patterns of scarabaeine. dung beetle diversity. Diversity and Distributions, 7: 161-174.

Davis, A. L. V., Scholtz, C. H. \& Philips, T. K., 2002. Historical biogeography of scarabaeine dung beetles. Journal of Biogeography, 29: 1217-1256.

Forgie, S., Philips, T. K. \& Scholtz, C. H., 2005. Evolution of the Scarabaeini (Scarabaeidae: Scarabaeinae). Systematic Entomology, 30: 60-96.

Halffter, G. \& Edmonds, W. D., 1982. The nesting behavior of dung beetles (Scarabaeinae): an ecological and evolutive approach. Instituto de Ecología, México Distrito Federal. México. 176 pp.

Hortal, J., 2004. Selección y Diseño de Áreas Prioritarias de Conservación de la Biodiversidad mediante Sinecología. Inventario y modelización predictiva de la distribución de los escarabeidos coprófagos (Coleoptera, scarabaeoidea) de Madrid. Tesis Doctoral, Universidad Autónoma de Madrid. 333 pp.

Howden, H. F., 1982. Larval and adult characters of Frickius Germain, its relationship to the Geotrupini, and a phylogeny of some major taxa in the Scarabaeoidea (Insecta: Coleoptera). Canadian Journal of Zoology, 60: 2713-2724.

Krell, F. T., 2000. The fossil record of Mesozoic and Tertiary Scarabaeoidea (Coleoptera: Polyphaga). Invertebrate Taxonomy, 14: 871-905.

Loвo, J. M., 2001. Decline of roller dung beetle (Scarabaeinae) populations in the Iberian peninsula during the $20^{\text {th }}$ century. Biological Conservation, 97: 43-50.

Lobo, J. M. \& Martín-Piera, F., 2002. Searching for a predictive model for species richness of Iberian dung beetles based on spatial and environmental variables. Conservation Biology, 16: 158-173.

Lobo, J. M., Verdú, J. R. \& Numa, C., 2006. Environmental and geographical factors affecting the Iberian distribution of flightless Jekelius species (Coleoptera:Geotrupidae). Diversity and Distributions, 12: 179-188.

López-Colón, J. I., 2000. Familia Geotrupidae. In: M. A. Ramos et al. (eds.). Fauna Ibérica, vol. 14, Coleoptera, Scarabaeoidea. Museo Nacional de Ciencias Naturales. CSIC. Madrid: 107-177. 
LuMARET, J.-P. \& LoBO, J. M., 1996. Geographic distribution of endemic dung beetles (Coleoptera, Scarabaeoidea) in the Western Palaearctic region. Biodiversity Letters, 3: 192-199.

Martín-PierA, F., 2000. Familia Scarabaeidae. In: M. A. Ramos et al. (eds.). Fauna Ibérica, vol. 14, Coleoptera, Scarabaeoidea. Museo Nacional de Ciencias Naturales. CSIC. Madrid: 207-432.

Martín-Piera, F. \& LóPez-Colón, J. I., 2000. Coleoptera, Scarabaeoidea I. En: Fauna Ibérica, vol. 14. Ramos, M. A. et al. (Eds.). Museo Nacional de Ciencias Naturales. CSIC. Madrid. 526 pp.

Montreuil, O., 1998. Analyse phylogénétique et paraphylie des Coprini et Dichotomiini (Coleoptera: Scarabaeidae). Scénario biogéographique. Annales de la Société Entomologique de France, 34: 135-148.

Palmer, M. \& CAMBefort, Y., 1997. Aptérisme et diversité dans le genre Thorectes Mulsant, 1842 (Coleoptera: Geotrupidae): Une étude phylogénétique et biogéographique des espèces méditerranéennes. Annales de la Société Entomologique de France, 33: 3-18.

PAlmer, M. \& CAMBefort, Y., 2000. Evidence for reticulate palaeogeography: beetle diversity linked to connection-disjunction cycles of the Gibraltar strait. Journal of Biogeography, 27: 403-416.

Philips, T. K., Edmonds, D. W. \& Scholtz, C. H., 2004b. Phylogenetic analysis of the New World Phanaeini (Scarabaeidae: Scarabaeinae): Hypotheses on origins and relationships. Insect Systematics and Evolution, 35: $43-63$

Philips, T. K., Pretorius, R. \& Scholtz, C. H., 2004a. A phylogenetic analysis of the dung beetles: (Scarabaeinae: Scarabaeidae): Unrolling an evolutionary history. Invertebrate Systematics, 18: 1-36.

Philips, T. K., Scholtz, C. H. \&. OcAmpo, F. C., 2002. A phylogenetic analysis of the Eucraniini (Scarabaeidae: Scarabaeinae). Insect Systematics and Evolution, 33: 241-252.

Scholtz, C. H. \& Chown, C. L., 1995. The evolution of habitat use and diet in the Scarabaeoidea: a phylogenetic approach. In: J. Pakaluk y S. A. Slipinski (eds.). Biology, Phylogeny, and Classification of Coleoptera. Papers Celebrating the $80^{\text {th }}$ Birthday of Roy A. Crowson. Muzeum I Instytut Zoologici PAN. Warszawa: 355-374.
Verdú, J. R. \& Galante, E., 2002. Climatic stress, food availability and human activity as determinants of endemism patterns in the Mediterranean region: the case of dung beetles (Coleoptera, Scarabaeoidea) in the Iberian Peninsula. Diversity and Distributions, 8: 259-274.

Verdú, J. R. \& Galante, E., 2004. Behavioural and morphological adaptations for a low-quality resource in semi-arid environments: dung beetles (Coleoptera, Scarabaeoidea) associated with the European rabbit (Oryctolagus cuniculus L.). Journal of Natural History, 38: 705-715.

Verdú, J. R., Galante, E. \& Lumaret, J. P., 1998. Description de la larve de Bolbelasmus bocchus (Erichson) et position systématique du genre (Coleoptera: Geotrupidae: Bolboceratinae). Annales de la Société Entomologique de France, 34: 245-251.

Verdú, J. R., Galante, E., Lumaret, J. P. \& CabreroSAÑUdO, F. J., 2004. Phylogenetic analysis of Geotrupidae (Coleoptera, Scarabaeoidea) based on larvae. Systematic Entomology, 29: 509-523.

Villalba, S., Lobo, J. M., Martín-Piera, F. \& ZARDOYA, R., 2002. Phylogenetic relationships of Iberian dung beetles (Coleoptera: Scarabaeinae): Insights on the evolution of nesting behaviour. Journal of Molecular Evolution, 55: 116-126.

ZuniNo, M., 1979. Gruppi artifiaciali e gruppi naturali negli Onthophagus (coleoptera, Scarabaeoidea). Bolletino del Museo Regionale di Scienze Naturali, Torino, 1: 1-18.

ZunINO, M., 1984a. Sistematica generica dei Geotrupinae (Coleoptera, Scarabaeoidea: Geotrupidae), filogenesi della sottofamiglia e considerazioni biogeografiche. Bolletino del Museo Regionale di Scienze Naturali, Torino, 2: 9-162.

ZuniNO, M., 1984b. Essai préliminaire sur l'évolution des armures génitales des Scarabaeinae, par rapport à la taxonomie du groupe et à l'évolution du comportement de nidification. Bulletin de la Société Entomologique de France, 88: 531-542.

Zunino, M., 1985. Las relaciones taxonómicas de los Phanaeina (Coleoptera, Scarabaeinae) y sus implicaciones biogeográficas. Folia Entomológica Mexicana, 64: 101-115. 